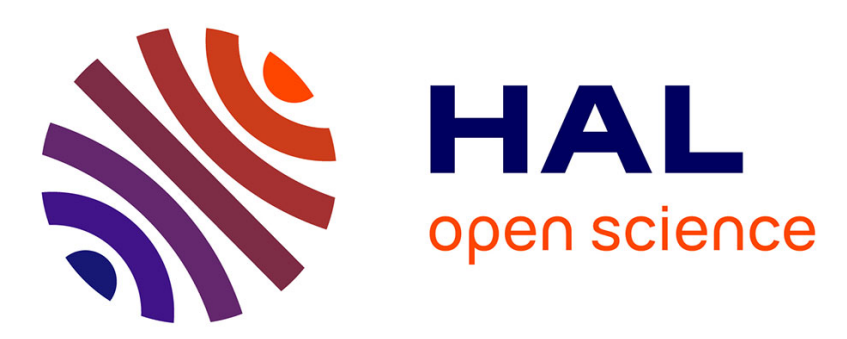

\title{
Exploiting partial or complete geometrical symmetry in 3D symmetric Galerkin indirect BEM formulations
}

Marc Bonnet

\section{To cite this version:}

Marc Bonnet. Exploiting partial or complete geometrical symmetry in 3D symmetric Galerkin indirect BEM formulations. International Journal for Numerical Methods in Engineering, 2003, 57, pp.10531083. 10.1002/nme.716 . hal-00092392

\section{HAL Id: hal-00092392 https://hal.science/hal-00092392}

Submitted on 9 Aug 2008

HAL is a multi-disciplinary open access archive for the deposit and dissemination of scientific research documents, whether they are published or not. The documents may come from teaching and research institutions in France or abroad, or from public or private research centers.
L'archive ouverte pluridisciplinaire HAL, est destinée au dépôt et à la diffusion de documents scientifiques de niveau recherche, publiés ou non, émanant des établissements d'enseignement et de recherche français ou étrangers, des laboratoires publics ou privés. 


\title{
Exploiting partial or complete geometrical symmetry in 3D symmetric Galerkin indirect BEM formulations.
}

\author{
Marc Bonnet \\ Laboratoire de Mécanique des Solides (UMR CNRS 7649), Ecole Polytechnique, Palaiseau, FRANCE
}

\begin{abstract}
SUMMARY
Procedures based on group representation theory, allowing the exploitation of geometrical symmetry in symmetric Galerkin BEM formulations, are investigated. In particular, this investigation is based on the weaker assumption of partial geometrical symmetry, where the boundary has two disconnected components, one of which is symmetric; this can be very useful for e.g. defect identification problems. The main development is expounded in the context of 3D Neumann elastostatic problems, considered as model problems; and then extended to SGBIE formulations for Dirichlet and/or scalar problems. Both Abelian and non-Abelian finite symmetry groups are considered. The effectiveness of the present approach is demonstrated through numerical examples, where both partial and complete symmetry are considered, in connection with both Abelian and non-Abelian symmetry groups. Copyright (c) 2003 John Wiley \& Sons, Ltd.
\end{abstract}

KEY WORDS: Symmetric Galerkin BEM, indirect BEM, geometrical symmetry

\section{INTRODUCTION}

When a linear boundary-value problem (BVP) exhibits geometrical symmetry, taking full advantage of it yields substantial computational benefits. In Bossavit [4], the linear representation theory for finite groups $[8,11]$ is shown to lead to the correct definition of (i) decomposition of function spaces into orthogonal subspaces of symmetric, skew-symmetric,... functions, and (ii) reconstruction of the global solution from these components; the (domain-based, FEM-oriented) weak formulation is thus recast into a block-diagonal form, each 'subproblem' being defined on a 'symmetry cell' (a subdomain of smallest measure that, under the action of the symmetry group, generates the entire initial domain) and associated to the corresponding projection of the boundary data. The procedure, being essentially an elaborate superposition technique, assumes linear constitutive properties. Similar principles are used by Allgower et al. [1] to block-diagonalize matrix equations resulting from discretization processes on geometrically symmetric domains.

The adaptation to boundary element methods (BEMs) of Bossavit's treatment is not straightforward, mostly because new boundaries are usually introduced along with the symmetry cell. This feature is unimportant in FEMs but clearly undesirable in BEMs, where subproblems should be stated only on

*Correspondence to: Laboratoire de Mécanique des Solides (UMR CNRS 7649), Ecole Polytechnique, F-91128 Palaiseau Cedex, FRANCE. bonnet@lms.polytechnique.fr 
symmetry cells of the original boundary. In an earlier work [2], this issue was adressed for collocation BEMs and Abelian (i.e. commutative) symmetry groups (see also [6]). Assuming standard methods to set up and solve the matrix equations, the theoretical computational gains (in relative terms, compared to using the same discretization without symmetry) were found to be $1 / n, 1 / n$ and $1 / n^{2}$ for the matrix storage requirement, matrix set-up time and solution time, respectively ( $n$ : number of elements in the symmetry group, e.g. $n=8$ for the group of symmetries with respect to three orthogonal planes).

This article is concerned with the formulation and numerical demonstration of procedures for exploiting geometrical symmetry in BEMs. The salient and novel features of the present investigation are as follows. Firstly, the exploitation of geometrical symmetry is considered here in the framework of symmetric Galerkin BEM (SGBEM) formulations. Secondly, procedures are developed for both Abelian and non-Abelian symmetry groups; as we will see, there are significant differences between these two cases. Thirdly, the approach is developed for the more general cases of partial geometrical symmetry, where the boundary has two (or more) disconnected components, one (or more) of which being invariant under a symmetry group. For instance, defect identification problems may involve bodies with external geometrical symmetry but containing internal cracks, voids, inclusions... of arbitrary shape and location. The formulations developed herein are expected to bring significant gains in computational efficiency by taking advantage of the fact that one component of the boundary (e.g. the external boundary in defect identification) exhibits geometrical symmetry.

The developments presented herein focus on the exploitation of symmetry in SGBEM formulations of Neumann or Dirichlet boundary-value problems (BVPs), the extension of the proposed treatment to SGBEM formulations of mixed BVPs being left out for a forthcoming investigation. For the sake of definiteness, the treatment is here fully developed in the framework of three-dimensional Neumann elastostatic BVPs, used as a convenient model situation. After the relevant governing equations are reviewed (section 2), the basic symmetry assumptions are discussed, and the concepts of complete or partial symmetry introduced, in section 3. Then, the symmetry-exploiting SGBEM formulation is established in sections 4 (for Abelian symmetry groups) and 5 (for non-Abelian symmetry groups), and modified in section 6 in order to circumvent all rigid-body eigensolutions that otherwise cause the bilinear operator to be non-invertible. Section 7 addresses the effect of symmetry exploitation on the interior displacement representation formula. Several important implementation issues are discussed in section 8. Section 9 addresses the straightforward adaptations of the proposed approach to Dirichlet elastostatic BVPs, scalar and/or complex-valued Neumann or Dirichlet BVPs. Finally, several numerical examples demonstrating the present strategy for three-dimensional Neumann elastostatic BVPs are presented in section 10.

\section{GOVERNING EQUATIONS}

In this paper, the use of geometrical symmetry is fully developed for the Neumann BVP of linear elastostatics, using a symmertic Galerkin formulation of the indirect integral equation based on representing the unknown displacement field by means of a double-layer elastic potential. This setting is chosen mainly as a representative model problem, and the developments to follow are directly adaptable to the SGBEM formulations of other scalar or vector linear BVPs, associated with either various other physical contexts (e.g. acoustics, elastodynamics, electromagnetics) or Dirichlet boundary conditions (in which case the main unknown field can be represented in terms of a singlelayer elastic potential), see section 9.

The displacement vector $\boldsymbol{u}$, strain tensor $\varepsilon$ and stress tensor $\boldsymbol{\sigma}$ in a three-dimensional isotropic

Int. J. Numer. Meth. Engng 2003; 57:1053-1083 
elastic medium $\Omega$, in the absence of body forces, are governed by the equilibrium, constitutive and compatibility field equations:

$$
\operatorname{div} \boldsymbol{\sigma}=\mathbf{0} \quad \boldsymbol{\sigma}=\mu\left[\frac{2 \nu}{1-2 \nu} \operatorname{Tr}(\varepsilon) \boldsymbol{I}+2 \varepsilon\right] \quad \boldsymbol{\varepsilon}=\left(\boldsymbol{\nabla} \boldsymbol{u}+\boldsymbol{\nabla}^{T} \boldsymbol{u}\right) / 2
$$

( $\mu$ : shear modulus, $\nu$ : Poisson ratio). Solutions to (1) are sought in the form of a double-layer elastic potential:

$$
u_{k}(\tilde{\boldsymbol{x}})=\int_{\partial \Omega} T_{i}^{k}(\tilde{\boldsymbol{x}}, \boldsymbol{x}) \phi_{i}(\boldsymbol{x}) \mathrm{d} S_{x}=:[\boldsymbol{A} \boldsymbol{\phi}](\tilde{\boldsymbol{x}})
$$

where the boundary $\partial \Omega$ is a bounded surface, either closed or open (or possibly a set of such surfaces) and $T_{i}^{k}(\tilde{\boldsymbol{x}}, \boldsymbol{x})$ denotes the $i$-th component of the traction vector associated with the Kelvin fundamental displacement $U_{i}^{k}(\tilde{\boldsymbol{x}}, \boldsymbol{x})$, i.e. the response at $\boldsymbol{x}$ of an unbounded three-dimensional elastic body to a unit point force applied at $\tilde{\boldsymbol{x}}$ in the direction $k$. The well-known expressions of $U_{i}^{k}$ and $T_{i}^{k}$ are:

$$
\begin{aligned}
U_{i}^{k}(\tilde{\boldsymbol{x}}, \boldsymbol{x}) & =\frac{1}{16 \pi \mu(1-\nu) r}\left[r_{, i} r_{, k}+(3-4 \nu) \delta_{i k}\right] \\
T_{i}^{k}(\tilde{\boldsymbol{x}}, \boldsymbol{x}) & =-\frac{1}{8 \pi(1-\nu) r^{2}}\left[3 r_{, i} r_{, k} r_{, j} n_{j}(\boldsymbol{x})+(1-2 \nu)\left(\delta_{i k} r_{, j} n_{j}(\boldsymbol{x})+r_{, i} n_{k}(\boldsymbol{x})-r_{, k} n_{i}(\boldsymbol{x})\right)\right]
\end{aligned}
$$

with $r=|\tilde{\boldsymbol{x}}-\boldsymbol{x}|$ and $r_{, a}=\partial r / \partial x_{a}=\left(x_{a}-\tilde{x}_{a}\right) / r$. The domain $\Omega$ may be either bounded or unbounded, with decay conditions at infinity implicitly enforced by the representation (2) in the latter case.

The unknown vector density $\phi$ does not usually have an immediately clear physical meaning, except when $\partial \Omega$ describes a crack surface (in which case $\phi$ is the crack opening displacement), and actual field variables must be recovered by means of the representation (2). To determine $\phi$ uniquely, boundary conditions must be specified. Integral representations of the form (2) are often used to formulate boundary integral equations (BIEs) for BVPs on domains bounded by $\partial \Omega$ with Neumann boundary data $\bar{p}$ prescribed on $S$. In particular, such problems lead to symmetric Galerkin BIE (SGBIE) formulations through a weighted-residual statement of the Neumann boundary condition:

$$
\int_{\partial \Omega} \boldsymbol{T}^{n}[\boldsymbol{A} \boldsymbol{\phi}](\tilde{\boldsymbol{x}}) \cdot \tilde{\boldsymbol{\phi}}^{\star}(\tilde{\boldsymbol{x}}) \mathrm{d} S_{\tilde{\boldsymbol{x}}}=\int_{\partial \Omega} \overline{\boldsymbol{p}}(\tilde{\boldsymbol{x}}) \cdot \tilde{\boldsymbol{\phi}}^{\star}(\tilde{\boldsymbol{x}}) \mathrm{d} S_{\tilde{x}} \quad\left(\forall \tilde{\boldsymbol{\phi}} \in \mathcal{U}=\left[H^{1 / 2}(S)\right]^{3}\right)
$$

where the traction vector operator $\boldsymbol{T}^{n} \boldsymbol{u}$ is defined by $\boldsymbol{T}^{n} \boldsymbol{u}=\boldsymbol{\sigma}(\boldsymbol{u}) \cdot \boldsymbol{n}, \tilde{\boldsymbol{\phi}}$ is a trial function and $z^{\star}$ denotes the complex conjugate of $z$. The operation $\left[\boldsymbol{T}^{n} \boldsymbol{A} \phi\right](\tilde{\boldsymbol{x}})$ gives rise to a hypersingular kernel involving a $r^{-3}$ singularity. After a well-documented regularization process [3, 7] involving two integrations by parts over $\partial \Omega$, the resulting SGBIE formulation, on which the present development is based, reads:

$$
\text { Find } \phi \in \mathcal{U}, \quad \mathcal{A}\left(\phi, \tilde{\phi}^{\star}\right)=\mathcal{L}\left(\tilde{\phi}^{\star}\right) \quad(\forall \tilde{\phi} \in \mathcal{U})
$$

In (6), the linear form $\mathcal{L}$ is defined by

$$
\mathcal{L}(\boldsymbol{v})=\langle\bar{p}, \boldsymbol{v}\rangle_{\partial \Omega}
$$

where $\langle,\rangle_{S}$ denotes the $L^{2}$ scalar product of vector functions on a generic surface $S$, i.e.:

$$
\langle\boldsymbol{f}, \boldsymbol{g}\rangle_{S}:=\int_{S} \boldsymbol{f}(\boldsymbol{x}) \cdot \boldsymbol{g}(\boldsymbol{x}) \mathrm{d} S_{x}
$$

while the symmetric bilinear form $\mathcal{A}$ is given by:

$$
\mathcal{A}\left(\boldsymbol{\phi}, \tilde{\boldsymbol{\phi}}^{\star}\right)=\int_{\partial \Omega} \int_{\partial \Omega} B_{i k q s}(\tilde{\boldsymbol{x}}, \boldsymbol{x}) R_{s} \phi_{k}(\boldsymbol{x}) R_{q} \tilde{\phi}_{i}^{\star}(\tilde{\boldsymbol{x}}) \mathrm{d} S_{x} \mathrm{~d} S_{\tilde{x}}
$$


In (9), the weakly singular kernel function $B_{i k q s}$ is given by:

$$
B_{i k q s}(\tilde{\boldsymbol{x}}, \boldsymbol{x})=\frac{\mu}{4 \pi(1-\nu) r}\left[\delta_{q s} r_{, i} r_{, k}+\delta_{i k} \delta_{q s}-2 \nu \delta_{i s} \delta_{q k}-(1-\nu) \delta_{i q} \delta_{k s}\right]
$$

and has symmetry properties which ensure the overall symmetry of $\mathcal{A}\left(\phi, \tilde{\phi}^{\star}\right)$ :

$$
B_{i k q s}(\tilde{\boldsymbol{x}}, \boldsymbol{x})=B_{i k q s}(\boldsymbol{x}, \tilde{\boldsymbol{x}})=B_{k i s q}(\tilde{\boldsymbol{x}}, \boldsymbol{x})
$$

Also, $R_{i} f$ denotes the $i$-th component of the surface curl of a scalar function $f$ [7] ( $e_{a b c}$ : permutation tensor):

$$
R_{i} f=e_{i j k} n_{j} f_{, k}
$$

Besides, if the surface $\partial \Omega$ is locally (e.g. element-wise) defined by a mapping $\Delta \subset \mathbb{R}^{2} \rightarrow \partial \Omega$, $\boldsymbol{\xi} \rightarrow \boldsymbol{x}(\boldsymbol{\xi})$, one has

$$
\left[R_{i} f\right](\boldsymbol{x}(\boldsymbol{\xi})) \mathrm{d} S_{x}=\left[\partial_{\xi_{1}} f \partial_{\xi_{2}} x_{i}-\partial_{\xi_{2}} f \partial_{\xi_{1}} x_{i}\right] \mathrm{d} \boldsymbol{\xi}
$$

which shows in particular that $R_{i} f$ is entirely determined by the local geometry of $\partial \Omega$ and the trace of $f$ on $\partial \Omega$, i.e. $R_{i}$ is a tangential differential operator.

Note that the SGBEM equation (6) involves complex conjugate of the trial density $\tilde{\phi}$, even though this may seem unnecessary for the model situation being considered, which is static (i.e. real-valued). The reason for choosing this setting will become apparent in sections 4 and 5 .

\section{SYMMETRY ASSUMPTIONS}

\subsection{Partial or complete geometrical symmetry}

The most important assumption for the present purposes is that the boundary $\partial \Omega$ has either partial or complete geometrical symmetry, in the following sense.

Partial symmetry. The assumption of partial symmetry postulates the existence of a finite group $\mathcal{S}=\left\{s_{1}, \ldots, s_{n}\right\}$ of $n$ isometries of $\mathbb{R}^{3}(n$ is the order of $\mathcal{S}$ ) and a partition of the boundary $\partial \Omega$ into two disconnected components $\Sigma, \Gamma$ such that $\Sigma$ is invariant under $\mathcal{S}$ whereas $\Gamma$ is not:

$$
\partial \Omega=\Sigma \cup \Gamma \quad s(\Sigma)=\Sigma(\forall s \in \mathcal{S})
$$

(recall that an isometry of $\mathbb{R}^{3}$ is a linear application $s: \mathbb{R}^{3} \rightarrow \mathbb{R}^{3}$ such that $|s \boldsymbol{x}|=|\boldsymbol{x}|$ $\left(\forall \boldsymbol{x} \in \mathbb{R}^{3}\right)$, where $|\boldsymbol{x}| \equiv(\boldsymbol{x} \cdot \boldsymbol{x})^{1 / 2}$ is the usual Euclidean norm in $\mathbb{R}^{3}$; hence each $s$ is represented by a $3 \times 3$ real-valued unitary matrix). The group operation is of course the composition of linear applications in $\mathbb{R}^{3}$, or, equivalently, the product of $3 \times 3$ unitary matrices. As an example, one of the simplest symmetry groups is $\mathcal{S}=\{I, s\}$ where $I$ is the identity in $\mathbb{R}^{3}$ and $s$ is a symmetry with respect to a plane (note that in this case $s^{-1}=s$ ). Some of the most common symmetry groups (plane, cyclic and dihedral symmetries) are listed in Appendix II.

For later convenience, the space $\mathcal{U}=\left[H^{1 / 2}(\partial \Omega)\right]^{3}$ is accordingly split into

$$
\mathcal{U}=\mathcal{U}_{\Sigma} \times \mathcal{U}_{\Gamma} \quad \text { with } \quad \mathcal{U}_{\Sigma}=\left[H^{1 / 2}(\Sigma)\right]^{3}, \mathcal{U}_{\Gamma}=\left[H^{1 / 2}(\Gamma)\right]^{3}
$$

One can then introduce a symmetry cell for $\Sigma$, i.e. a subset $C$ of $\Sigma$ such that

$$
\operatorname{Area}(C)=\operatorname{Area}(\Sigma) / n, \quad \Sigma=s_{1}(C) \cup \ldots \cup s_{n}(C)
$$

For example, $\Sigma$ is the (symmetric) external boundary while $\Gamma$ is a (collection of) interior hole(s) or $\operatorname{crack}(\mathrm{s})$ of arbitrary shape and location. 
Complete symmetry. This refers to the special partial symmetry cases where $\Sigma=\partial \Omega$ and $\Gamma=\emptyset$, i.e. where the whole boundary $\partial \Omega$ (and hence also $\Omega$ ) is invariant under $\mathcal{S}$.

Exploiting partial or complete geometrical symmetry in the SGBEM formulation (6) will involve mapping each $s(C)$ onto $C$ by $\boldsymbol{x} \in C \rightarrow \boldsymbol{z}=s \boldsymbol{x} \in s(C)$ and express integrals over $\Sigma$ as sums of integrals over $C$. In particular, since $s$ is an isometry, one always has:

$$
\mathrm{d} S(s \boldsymbol{x})=\mathrm{d} S(\boldsymbol{x}) \quad \boldsymbol{n}(s \boldsymbol{x})=s[\boldsymbol{n}(\boldsymbol{x})]
$$

The resulting matrix operators produced by the discretization process are thus expected to be of smaller size than those corresponding to the integral equations discretized without using symmetry. Note that no symmetry is assumed regarding the Neumann data $\bar{p}$.

\subsection{Material symmetry: equivariance}

In addition to geometrical symmetry, one must assume that the material properties are also invariant under the symmetry group $\mathcal{S}$. Accordingly, a bilinear form $\mathcal{A}(\boldsymbol{u}, \boldsymbol{v})$ over $\mathcal{U}_{\Sigma} \times \mathcal{U}_{\Sigma}$ is said to have the equivariance property if [4]:

$$
\mathcal{A}\left(\mathcal{T}_{s} \boldsymbol{u}, \mathcal{T}_{s} \boldsymbol{v}\right)=\mathcal{A}(\boldsymbol{u}, \boldsymbol{v}) \quad\left(\forall s \in \mathcal{S}, \forall \boldsymbol{u}, \boldsymbol{v} \in \mathcal{U}_{\Sigma}\right)
$$

where the linear operator $\mathcal{T}_{s}$, which represents the action of $s \in \mathcal{S}$ on a vector function, is defined by

$$
\mathcal{T}_{s} \boldsymbol{u}(\boldsymbol{x})=s \boldsymbol{u}\left(s^{-1} \boldsymbol{x}\right)
$$

Here, from $\left(14_{2}\right)$ and the identities:

$$
\boldsymbol{r}(s \tilde{\boldsymbol{x}}, s \boldsymbol{x})=s \boldsymbol{r}(\tilde{\boldsymbol{x}}, \boldsymbol{x}) \quad r(s \tilde{\boldsymbol{x}}, s \boldsymbol{x})=r(\tilde{\boldsymbol{x}}, \boldsymbol{x})
$$

(where $\boldsymbol{r}(\tilde{\boldsymbol{x}}, \boldsymbol{x}) \equiv \boldsymbol{x}-\tilde{\boldsymbol{x}}$ and $r(\tilde{\boldsymbol{x}}, \boldsymbol{x})=|\tilde{\boldsymbol{x}}, \boldsymbol{x}|)$, it is easy to check that:

$$
\begin{aligned}
& B_{i k q s}(\tilde{\boldsymbol{x}}, \boldsymbol{x})=s_{i j} s_{k \ell} s_{q r} s_{s t} B_{j \ell r t}\left(s^{-1} \tilde{\boldsymbol{x}}, s^{-1} \boldsymbol{x}\right) \\
& \left\{R_{i}\left[\mathcal{T}_{s} u\right]_{k} \mathrm{~d} S\right\}(\boldsymbol{x})=s_{i j} s_{k \ell}\left\{R_{j} u_{\ell} \mathrm{d} S\right\}\left(s^{-1} \boldsymbol{x}\right)
\end{aligned}
$$

Then, the bilinear form defined by (9) is easily seen to verify (15) by virtue of $\left(14_{1}\right)$ and the fact that $s^{-1}=s^{T}$, i.e. $s_{i a} s_{i b}=\delta_{a b}$, holds for any isometry. Similarly, the kernels $U_{i}^{k}$ and $T_{i}^{k}$ have from $\left(14_{2}\right)$ and (17) the following equivariance properties

$$
U_{i}^{k}(s \tilde{\boldsymbol{x}}, s \boldsymbol{x})=s_{i j} s_{k \ell} U_{j}^{\ell}(\tilde{\boldsymbol{x}}, \boldsymbol{x}) \quad T_{i}^{k}(s \tilde{\boldsymbol{x}}, s \boldsymbol{x})=s_{i j} s_{k \ell} T_{j}^{\ell}(\tilde{\boldsymbol{x}}, \boldsymbol{x})
$$

One can also easily show from (18) that, for any $(s, \tilde{s}) \in \mathcal{S} \times \mathcal{S}$

$$
B_{i k q s}(\tilde{s} \tilde{\boldsymbol{x}}, s \boldsymbol{x}) R_{s}[s \phi]_{k}(\boldsymbol{x}) R_{q}[\tilde{s} \tilde{\phi}]_{i}^{\star}(\tilde{\boldsymbol{x}})=B_{i k q s}(\tilde{\boldsymbol{x}}, t \boldsymbol{x}) R_{s}[t \phi]_{k}(\boldsymbol{x}) R_{q} \tilde{\phi}_{i}^{\star}(\tilde{\boldsymbol{x}}) \quad\left(t=\tilde{s}^{-1} s\right)
$$

\section{EXPLOITING GEOMETRICAL SYMMETRY: THE ABELIAN CASE}

The methodology developed in this article is based on results from the theory of linear representations of finite groups. Abelian (or commutative, i.e. $\forall s, t \in \mathcal{S}, s t=t s$ ) and non-Abelian symmetry groups lead to quite different formulations. In this section, $\mathcal{S}$ is an Abelian finite group of order $n$; this includes the common cases of groups of symmetries w.r.t. $k$ orthogonal planes (with $n=2^{k}$ and $1 \leq k \leq 3$ ) and the group $C_{n}=\left\{\mathrm{I}, r, r^{2}, \ldots, r^{n-1}\right\}$ of cyclic symmetry generated by a rotation $r$ of angle $2 \pi / n$, with $2 \leq n$ ). The more involved non-Abelian case is deferred to section 5 . 


\subsection{Linear representations of finite Abelian groups}

To exploit symmetry in a systematic way, it is first necessary to gather some definitions and results from the theory of linear representations of finite groups $[4,8,11]$.

Any finite Abelian group $\mathcal{S}$ of order $n$ possesses $n$ irreducible linear representations, i.e. $n$ applications $\rho_{\nu}: \mathcal{S} \rightarrow \mathbb{C}$ having the following properties:

$$
\left|\rho_{\nu}(s)\right|=1 \quad \rho_{\nu}(s t)=\rho_{\nu}(s) \rho_{\nu}(t) \quad \rho_{\nu}\left(s^{-1}\right)=\rho_{\nu}^{\star}(s)
$$

for any $s, t \in \mathcal{S}, 1 \leq \nu \leq n$, as well as the orthogonality property:

$$
\frac{1}{n} \sum_{s \in \mathcal{S}} \rho_{\nu}(s) \rho_{\mu}^{\star}(s)=\delta_{\mu \nu}
$$

Hence, the two-dimensional array $\left[\rho_{\nu}(s)\right]_{s \in \mathcal{S}, 1 \leq \nu \leq n}$ is a unitary matrix of $\mathbb{C}^{n, n}$ and the following orthogonality property holds as well:

$$
\frac{1}{n} \sum_{\nu=1}^{n} \rho_{\nu}(s) \rho_{\nu}^{\star}(t)=\delta_{s t}
$$

The $\rho_{\nu}$ are known for all usual groups, see Appendix II and in particular Table $\mathrm{X}$ for the groups $P_{1}, P_{12}, P_{123}$ and Eq. (88) for the groups $C_{n}$.

The $\rho_{\nu}: \mathcal{S} \rightarrow \mathbb{C}$ can be viewed as a group isomorphism between $\mathcal{S}$ and $\operatorname{GL}(\mathbb{C})$, the multiplicative group of linear endomorphisms of $\mathbb{C}$, and are said to be of degree one. In contrast, when $\mathcal{S}$ is not Abelian, some of the irreducible representations are necessarily of degree $\geq 2$.

The linear operators $\boldsymbol{P}_{\nu}$ defined by:

$$
\boldsymbol{\varphi} \rightarrow\left[\boldsymbol{P}_{\nu} \boldsymbol{\varphi}\right](\boldsymbol{x})=\frac{1}{n} \sum_{t \in \mathcal{S}} \rho_{\nu}^{\star}(t)\left[\mathcal{T}_{t} \boldsymbol{\varphi}\right](\boldsymbol{x})=\frac{1}{n} \sum_{t \in \mathcal{S}} \rho_{\nu}^{\star}(t) t \boldsymbol{\varphi}\left(t^{-1} \boldsymbol{x}\right)
$$

are readily shown using (23) to be orthogonal projectors for the scalar product of $L^{2}(\Sigma)$, i.e.:

$$
\left\langle\boldsymbol{P}_{\mu} \boldsymbol{\varphi},\left(\boldsymbol{P}_{\nu} \boldsymbol{\varphi}\right)^{\star}\right\rangle_{\Sigma}=0 \quad \mu \neq \nu
$$

Then, by virtue of (24), any vector function $\varphi$ defined on $\Sigma$ admits, as shown in Appendix I, the decomposition:

$$
\boldsymbol{\varphi}=\sum_{\nu=1}^{n} \boldsymbol{P}_{\nu} \boldsymbol{\varphi}
$$

Let $\varphi_{\nu}$ denote the restriction to $C$ of $\boldsymbol{P}_{\nu} \boldsymbol{\varphi}$. Then, from the properties (22), it is easy to show that, for any $\boldsymbol{x} \in C$ and any $s \in \mathcal{S}$ :

$$
\left[\boldsymbol{P}_{\nu} \boldsymbol{\varphi}\right](s \boldsymbol{x})=\rho_{\nu}\left(s^{-1}\right) s \boldsymbol{\varphi}_{\nu}(\boldsymbol{x})
$$

If $\boldsymbol{x}$ is an interior point of $C$, then $s \boldsymbol{x} \notin C$ if $s \neq I$ (otherwise, $C$ would not be a symmetry cell). On the other hand, if $\boldsymbol{x}$ is located on the edge of $C$ (i.e. $\boldsymbol{x} \in \partial C$ ), it is possible to find $s \in \mathcal{S}, s \neq I$ such that $s \boldsymbol{x} \in \partial C$. Thus, for any $\boldsymbol{x} \in \partial C$, let

$$
\mathcal{I}(\boldsymbol{x})=\{s \in \mathcal{S}, s \boldsymbol{x} \in \partial C\}
$$

Then, identity (27) implies that the $\varphi_{\nu}$ obtained from a given $\varphi \in \mathcal{U}_{\Sigma}$ must satisfy at any $\boldsymbol{x} \in \partial C$ the constraints:

$$
\boldsymbol{\varphi}_{\nu}(s \boldsymbol{x})-\rho_{\nu}\left(s^{-1}\right) s \boldsymbol{\varphi}_{\nu}(\boldsymbol{x})=0 \quad(\forall \boldsymbol{x} \in \partial C, \forall s \in \mathcal{I}(\boldsymbol{x}))
$$


Let $\mathcal{V}_{\nu}$ denote the set of vector functions defined on $C$ for which (29) holds for a given $\nu$. When $\mathcal{I}(\boldsymbol{x})=\{I\}$, the constraint (29) becomes trivial, as expected.

Consequently, for any $\boldsymbol{x} \in C$ and any $s \in \mathcal{S}$, the value $\varphi(s \boldsymbol{x})$ of any vector function $\varphi \in \mathcal{U}_{\Sigma}$ at the image $s \boldsymbol{x}$ of $\boldsymbol{x}$ (i.e. at any point of $\Sigma$ ) can be decomposed into a weighted sum of the $n$ functions $\varphi_{\nu} \in \mathcal{V}_{\nu}$ defined on the symmetry cell:

$$
\boldsymbol{\varphi}(s \boldsymbol{x})=\sum_{\nu=1}^{n} \rho_{\nu}\left(s^{-1}\right) s \boldsymbol{\varphi}_{\nu}(\boldsymbol{x})
$$

The main idea behind the development to follow is to find governing SGBEM formulations for the $n$ unknown functions $\varphi_{\nu}$, each defined on the symmetry cell $C$, knowing that the solution to the original SGBEM equations can then be reconstructed at any point of the original surface $\Sigma$ by equation (30).

\subsection{Exploitation of partial geometrical symmetry}

Under the assumption of partial geometrical symmetry, one can map each $s(C)$ onto $C$ by $\boldsymbol{x} \in C \rightarrow$ $\boldsymbol{z}=s \boldsymbol{x} \in s(C)$ and express integrals over $\Sigma$ as sums of integrals over $C$, with the help of the identities (14). In particular, the bilinear form $\mathcal{A}\left(\phi, \tilde{\phi}^{\star}\right)$ and linear form $\mathcal{L}\left(\tilde{\phi}^{\star}\right)$ defined by (9) and (7) take the form:

$$
\begin{aligned}
\mathcal{A}\left(\boldsymbol{\phi}, \tilde{\boldsymbol{\phi}}^{\star}\right) & =\mathcal{B}\left(\boldsymbol{\varphi}, \tilde{\varphi}^{\star}\right)+\mathcal{C}\left(\boldsymbol{\psi}, \tilde{\varphi}^{\star}\right)+\mathcal{C}\left(\tilde{\boldsymbol{\psi}}^{\star}, \boldsymbol{\varphi}\right)+\mathcal{D}\left(\boldsymbol{\psi}, \tilde{\boldsymbol{\psi}}^{\star}\right) \\
\mathcal{L}\left(\tilde{\boldsymbol{\phi}}^{\star}\right) & =\mathcal{F}\left(\tilde{\boldsymbol{\varphi}}^{\star}\right)+\mathcal{G}\left(\tilde{\boldsymbol{\psi}}^{\star}\right)
\end{aligned}
$$

where $\boldsymbol{\varphi}, \tilde{\varphi} \in \mathcal{U}_{\Sigma}=\left[H^{1 / 2}(\Sigma)\right]^{3}$ and $\boldsymbol{\psi}, \tilde{\boldsymbol{\psi}} \in \mathcal{U}_{\Gamma}=\left[H^{1 / 2}(\Gamma)\right]^{3}$ denote the restrictions of $\phi, \tilde{\phi}$ on $\Sigma$ and on $\Gamma$, respectively, and with

$$
\begin{aligned}
\mathcal{B}\left(\boldsymbol{\varphi}, \tilde{\boldsymbol{\varphi}}^{\star}\right) & =\sum_{s \in \mathcal{S}} \sum_{\tilde{s} \in \mathcal{S}} \int_{C} \int_{C} B_{i k q s}(\tilde{s} \tilde{\boldsymbol{x}}, s \boldsymbol{x}) R_{s} \varphi_{k}(s \boldsymbol{x}) R_{q} \tilde{\varphi}_{i}^{\star}(\tilde{s} \tilde{\boldsymbol{x}}) \mathrm{d} S_{x} \mathrm{~d} S_{\tilde{x}} \\
\mathcal{C}\left(\boldsymbol{\psi}, \tilde{\boldsymbol{\varphi}}^{\star}\right) & =\sum_{\tilde{s} \in \mathcal{S}} \int_{C} \int_{\Gamma} B_{i k q s}(\tilde{\boldsymbol{s}} \tilde{\boldsymbol{x}}, \boldsymbol{x}) R_{s} \psi_{k}(\boldsymbol{x}) R_{q} \tilde{\varphi}_{i}^{\star}(\tilde{s} \tilde{\boldsymbol{x}}) \mathrm{d} S_{x} \mathrm{~d} S_{\tilde{x}} \\
\mathcal{D}\left(\boldsymbol{\psi}, \tilde{\boldsymbol{\psi}}^{\star}\right) & =\int_{\Gamma} \int_{\Gamma} B_{i k q s}(\tilde{\boldsymbol{x}}, \boldsymbol{x}) R_{s} \psi_{k}(\boldsymbol{x}) R_{q} \tilde{\psi}_{i}^{\star}(\tilde{\boldsymbol{x}}) \mathrm{d} S_{x} \mathrm{~d} S_{\tilde{x}}
\end{aligned}
$$

and

$$
\mathcal{F}\left(\tilde{\boldsymbol{\varphi}}^{\star}\right)=\sum_{\tilde{s} \in \mathcal{S}} \int_{C} \overline{\boldsymbol{p}}(\tilde{s} \tilde{\boldsymbol{x}}) \cdot \tilde{\boldsymbol{\varphi}}^{\star}(\tilde{\boldsymbol{s}} \tilde{\boldsymbol{x}}) \mathrm{d} S_{\tilde{x}} \quad \mathcal{G}\left(\tilde{\boldsymbol{\psi}}^{\star}\right)=\left\langle\overline{\boldsymbol{p}}, \tilde{\boldsymbol{\psi}}^{\star}\right\rangle_{\Gamma}
$$

Now, inserting the decomposition (30) for both $\varphi$ and $\tilde{\varphi}$ in $\mathcal{B}\left(\varphi, \tilde{\varphi}^{\star}\right)$ defined by (33), one has:

$$
\mathcal{B}\left(\boldsymbol{\varphi}, \tilde{\boldsymbol{\varphi}}^{\star}\right)=\sum_{\mu=1}^{n} \sum_{\nu=1}^{n} \sum_{s \in \mathcal{S}} \sum_{\tilde{s} \in \mathcal{S}} \rho_{\nu}(\tilde{s}) \rho_{\mu}\left(s^{-1}\right) \int_{C} \int_{C} B_{i k q s}(\tilde{s} \tilde{\boldsymbol{x}}, s \boldsymbol{x}) R_{s}\left[s \varphi_{\nu}\right]_{k}(\boldsymbol{x}) R_{q}\left[\tilde{s} \tilde{\varphi}_{\mu}\right]_{i}^{\star}(\tilde{\boldsymbol{x}}) \mathrm{d} S_{x} \mathrm{~d} S_{\tilde{x}}
$$

where property (22) has been used. Next, using the change of variable $t=\tilde{s}^{-1} s$ (i.e. $s=\tilde{s} t$ ) together with equivariance (21), one gets:

$$
\mathcal{B}\left(\boldsymbol{\varphi}, \tilde{\boldsymbol{\varphi}}^{\star}\right)=\sum_{\mu=1}^{n} \sum_{\nu=1}^{n} \sum_{t \in \mathcal{S}} \sum_{\tilde{s} \in \mathcal{S}} \rho_{\nu}(\tilde{s}) \rho_{\mu}\left(t^{-1}\right) \rho_{\mu}\left(\tilde{s}^{-1}\right) \mathcal{B}_{t}\left(\boldsymbol{\varphi}_{\nu}, \tilde{\boldsymbol{\varphi}}_{\mu}^{\star}\right)
$$


having put

$$
\mathcal{B}_{t}\left(\boldsymbol{\varphi}, \tilde{\boldsymbol{\varphi}}^{\star}\right)=\int_{C} \int_{C} B_{i k q s}(\tilde{\boldsymbol{x}}, t \boldsymbol{x}) R_{s}[t \boldsymbol{\varphi}]_{k}(\boldsymbol{x}) R_{q} \tilde{\varphi}_{i}^{\star}(\tilde{\boldsymbol{x}}) \mathrm{d} S_{x} \mathrm{~d} S_{\tilde{x}}
$$

Then, by virtue of the orthogonality property (23):

$$
\begin{aligned}
\mathcal{B}\left(\boldsymbol{\varphi}, \tilde{\boldsymbol{\varphi}}^{\star}\right) & =\sum_{\mu=1}^{n} \sum_{\nu=1}^{n} \sum_{t \in \mathcal{S}} \rho_{\mu}\left(t^{-1}\right)\left\{\sum_{\tilde{s} \in \mathcal{S}} \rho_{\nu}(\tilde{s}) \rho_{\mu}\left(\tilde{s}^{-1}\right)\right\} \mathcal{B}_{t}\left(\boldsymbol{\varphi}_{\nu}, \tilde{\boldsymbol{\varphi}}_{\mu}^{\star}\right) \\
& =\sum_{\nu=1}^{n}\left\{n \sum_{t \in \mathcal{S}} \rho_{\nu}\left(t^{-1}\right) \mathcal{B}_{t}\left(\boldsymbol{\varphi}_{\nu}, \tilde{\boldsymbol{\varphi}}_{\nu}^{\star}\right)\right\} \\
& \equiv \sum_{\nu=1}^{n} \mathcal{B}_{\nu}\left(\boldsymbol{\varphi}_{\nu}, \tilde{\boldsymbol{\varphi}}_{\nu}^{\star}\right)
\end{aligned}
$$

The bilinear form $\mathcal{B}\left(\varphi, \tilde{\varphi}^{\star}\right)$ is thus seen to have been reduced to a block-diagonal form.

One establishes in a similar way the decompositions:

$$
\mathcal{C}\left(\boldsymbol{\psi}, \tilde{\boldsymbol{\varphi}}^{\star}\right)=\sum_{\nu=1}^{n} \mathcal{C}_{\nu}\left(\boldsymbol{\psi}, \tilde{\boldsymbol{\varphi}}_{\nu}^{\star}\right) \quad \mathcal{F}\left(\tilde{\boldsymbol{\varphi}}^{\star}\right)=\sum_{\nu=1}^{n} \mathcal{F}_{\nu}\left(\tilde{\boldsymbol{\varphi}}_{\nu}^{\star}\right)
$$

with

$$
\begin{aligned}
\mathcal{C}_{\nu}\left(\boldsymbol{\psi}, \tilde{\boldsymbol{\varphi}}_{\nu}^{\star}\right) & =\sum_{\tilde{s} \in \mathcal{S}} \rho_{\nu}(\tilde{s}) \int_{C} \int_{\Gamma} B_{i k q s}(\tilde{s} \tilde{\boldsymbol{x}}, \boldsymbol{x}) R_{s} \psi_{k}(\boldsymbol{x}) R_{q}\left[\tilde{s} \tilde{\varphi}_{\nu}\right]_{i}^{\star}(\tilde{\boldsymbol{x}}) \mathrm{d} S_{x} \mathrm{~d} S_{\tilde{x}} \\
\mathcal{F}_{\nu}\left(\tilde{\boldsymbol{\varphi}}_{\nu}^{\star}\right) & =n\left\langle\overline{\boldsymbol{p}}_{\nu}, \tilde{\boldsymbol{\varphi}}_{\nu}^{\star}\right\rangle_{C}
\end{aligned}
$$

Gathering results (37) and (38), the initial integral equation (6) finally reduces to a set of SGBIE problems of the form:

Find $\varphi_{\nu} \in \mathcal{V}_{\nu}, \boldsymbol{\psi} \in \mathcal{U}_{\Gamma} ; \forall \tilde{\varphi}_{\nu} \in \mathcal{V}_{\nu}, \tilde{\boldsymbol{\psi}} \in \mathcal{U}_{\Gamma}$

$$
\left\{\begin{aligned}
\mathcal{B}_{\nu}\left(\boldsymbol{\varphi}_{\nu}, \tilde{\boldsymbol{\varphi}}_{\nu}^{\star}\right)+\mathcal{C}_{\nu}\left(\boldsymbol{\psi}, \tilde{\boldsymbol{\varphi}}_{\nu}^{\star}\right) & =\mathcal{F}_{\nu}\left(\tilde{\boldsymbol{\varphi}}_{\nu}^{\star}\right) \quad(1 \leq \nu \leq n) \\
\sum_{\nu=1}^{n} \mathcal{C}_{\nu}\left(\tilde{\boldsymbol{\psi}}^{\star}, \boldsymbol{\varphi}_{\nu}\right)+\mathcal{D}\left(\boldsymbol{\psi}, \tilde{\boldsymbol{\psi}}^{\star}\right) & =\mathcal{G}\left(\tilde{\boldsymbol{\psi}}^{\star}\right)
\end{aligned}\right.
$$

\subsection{Symmetry properties of the formulation (39).}

The irreducible representations $\rho_{\nu}$ may be complex-valued (e.g. (88) for cyclic groups). It can be shown that any complex-valued $\rho_{\nu}$ can be associated by conjugate pairs, i.e. that there exists $\nu^{\star}$ such that $\rho_{\nu^{\star}}(\mathcal{S})=\rho_{\nu}^{\star}(\mathcal{S})$ (with $\nu^{\star}=\nu$ if $\rho_{\nu}$ is real-valued). Thus, Eq. (29) implies that $\boldsymbol{v} \in \mathcal{V}_{\nu} \Rightarrow \boldsymbol{v}^{\star} \in \mathcal{V}_{\nu^{\star}}$. Besides, a useful consequence of equivariance (21) and the symmetry properties (10) of the kernel $B_{i k q s}$ is:

$$
\mathcal{B}_{t}(\boldsymbol{u}, \boldsymbol{v})=\mathcal{B}_{t^{-1}}(\boldsymbol{v}, \boldsymbol{u})
$$

which in turn implies that:

$$
\mathcal{B}_{\nu}(\boldsymbol{u}, \boldsymbol{v})=\mathcal{B}_{\nu^{\star}}(\boldsymbol{v}, \boldsymbol{u}) \quad \text { complex-valued } \rho_{\nu}
$$

i.e. that, although $\mathcal{B}$ is symmetric, the $\mathcal{B}_{\nu}(\boldsymbol{u}, \boldsymbol{v})$ are not individually symmetric, but have a 'reciprocal symmetry'. In some cases, including the very common one of symmetry with respect to coordinate planes, the $\rho_{\nu}$ are real-valued (see table X); then, $\boldsymbol{v} \in \mathcal{V}_{\nu} \Rightarrow \boldsymbol{v}^{\star} \in \mathcal{V}_{\nu}$ and the $\mathcal{B}_{\nu}$ are then symmetric:

$$
\mathcal{B}_{\nu}(\boldsymbol{u}, \boldsymbol{v})=\mathcal{B}_{\nu}(\boldsymbol{v}, \boldsymbol{u}) \quad \text { real-valued } \rho_{\nu}
$$




\section{EXPLOITING GEOMETRICAL SYMMETRY: THE NON-ABELIAN CASE}

In this section, $\mathcal{S}$ is a non-Abelian finite group of order $n$, i.e. there exist $s, t \in \mathcal{S}$ such that $s t \neq t s$. This includes the important practical case of the dihedral symmetry group $D_{k}$, i.e. the group of order $n=2 k$ of the affine transformations that leave a regular $k$-gon unchanged (see Figure 1 for an illustration of $\left.D_{5}\right)$. Again, some useful mathematical definitions and results $[4,8,11]$ need first to be gathered.

\subsection{Linear representations of finite non-Abelian groups}

Irreducible representations $\rho_{\nu}$ of $\mathcal{S}$ can again be defined, but this time they have integer degree $d_{\nu} \geq 1$ :

$$
\rho_{\nu}: s \in \mathcal{S} \rightarrow \rho_{\nu}(s) \in \mathrm{GL}\left(\mathbb{C}^{d_{\nu}}\right)
$$

i.e. each $\rho_{\nu}(s)$ is a linear endomorphism of a $d_{\nu}$-dimensional complex vector space; besides, each $\rho_{\nu}(s)$ is unitary, i.e.:

$$
\left[\rho_{\nu}(s)\right]^{-1}=\rho_{\nu}^{\star}(s) \quad(\forall s \in \mathcal{S})
$$

and at least one of them has degree $d_{\nu} \geq 2$. Moreover, the number $m$ of such representations and their degrees $d_{\nu}$ are such that:

$$
\sum_{\nu=1}^{m} d_{\nu}^{2}=n
$$

Besides, since the $3 \times 3$ unitary matrices associated to the elements of $\mathcal{S}$ themselves define representations of $\mathcal{S}$ (not necessarily irreducible), it follows that the irreducible representations of isometry groups of the physical space $\mathbb{R}^{3}$ necessarily have degree $\leq 3$. The irreducible representations of dihedral groups are given in Appendix II.

The properties of the irreducible representations $\rho_{\nu}$ include the preservation of group structure:

$$
\rho_{\nu}^{a b}(s t)=\sum_{c=1}^{d_{\nu}} \rho_{\nu}^{a c}(s) \rho_{\nu}^{c b}(t) \quad(\forall s, t \in \mathcal{S}) \quad \rho_{\nu}^{a b}(I)=\delta_{a b}
$$

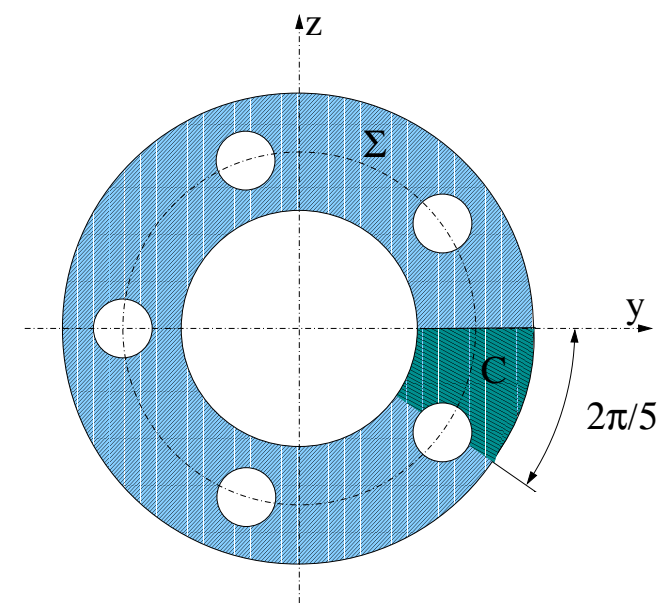

Figure 1. Illustration of the dihedral symmetry group $D_{5}$ (top view of the cylinder with five channels of Section 10.5). 
which implies in particular, since $\rho_{\nu}$ is unitary, that:

$$
\rho_{\nu}^{a b}\left(s^{-1}\right)=\left[\rho_{\nu}^{b a}(s)\right]^{\star}
$$

and the orthogonality relation:

$$
\frac{d_{\nu}}{n} \sum_{s \in \mathcal{S}} \rho_{\nu}^{a b}(s)\left[\rho_{\mu}^{c d}(s)\right]^{\star}=\delta_{a c} \delta_{b d} \delta_{\mu \nu}
$$

which in turn implies the 'dual' orthogonality relation:

$$
\sum_{\nu=1}^{m} \sum_{a, b=1}^{d_{\nu}} \frac{d_{\nu}}{n}\left[\rho_{\mu}^{a b}(s)\right]^{\star} \rho_{\nu}^{a b}(t)=\delta_{s t}
$$

Introduce the linear operators $\boldsymbol{P}_{\nu}^{a b}$ defined by:

$$
\left[\boldsymbol{P}_{\nu}^{a b} \boldsymbol{\varphi}\right](\boldsymbol{x})=\frac{d_{\nu}}{n} \sum_{t \in \mathcal{S}} \rho_{\nu}^{\star a b}(t)\left[\mathcal{T}_{t} \boldsymbol{\varphi}\right](\boldsymbol{x})=\frac{d_{\nu}}{n} \sum_{t \in \mathcal{S}} \rho_{\nu}^{\star a b}(t) t \boldsymbol{\varphi}\left(t^{-1} \boldsymbol{x}\right)
$$

The $\boldsymbol{P}_{\nu}^{a a}$ are readily shown using (46) to be orthogonal projectors for the scalar product of $L^{2}(\Sigma)$, i.e.:

$$
\left\langle\boldsymbol{P}_{\mu}^{a a} \boldsymbol{\varphi},\left(\boldsymbol{P}_{\nu}^{a a} \boldsymbol{\varphi}\right)^{\star}\right\rangle_{\Sigma}=0 \quad \mu \neq \nu
$$

Then, by virtue of (47), any vector function $\varphi$ defined on $\Sigma$ admits, as shown in Appendix I, the decomposition:

$$
\boldsymbol{\varphi}=\sum_{\nu=1}^{n} \sum_{a=1}^{d_{\nu}} \boldsymbol{P}_{\nu}^{a a} \boldsymbol{\varphi}
$$

Let $\varphi_{\nu}^{a b}$ denote the restriction on $C$ of the projection $\boldsymbol{P}_{\nu}^{a b} \boldsymbol{\varphi}$. Then, from definition (48) and the properties of the representations, one has for any $s \in \mathcal{S}$ and $\varphi \in \mathcal{U}_{\Sigma}$ :

$$
\left[\boldsymbol{P}_{\nu}^{a b} \boldsymbol{\varphi}\right](s \boldsymbol{x})=\sum_{c=1}^{d_{\nu}} \rho_{\nu}^{c a}\left(s^{-1}\right) s \boldsymbol{\varphi}_{\nu}^{c b}(\boldsymbol{x})
$$

Moreover, it is easy to show, from (50), that the $d_{\nu}$-uple $\left\{\boldsymbol{\varphi}_{\nu}^{a b}, 1 \leq a \leq d_{\nu}\right\}$ of functions defined on $C$ are subject to the following constraints:

$$
\boldsymbol{\varphi}_{\nu}^{a b}(s \boldsymbol{x})-\sum_{c=1}^{d_{\nu}} \rho_{\nu}^{c a}\left(s^{-1}\right) s \boldsymbol{\varphi}_{\nu}^{c b}(\boldsymbol{x})=\mathbf{0} \quad(\forall \boldsymbol{x} \in \partial C, \forall s \in \mathcal{I}(\boldsymbol{x}))
$$

(note that the constraint does not depend on the rightmost index $b$ ), where $\mathcal{I}(\boldsymbol{x})$ is again defined by (28). Accordingly, for the non-Abelian case, let $\mathcal{V}_{\nu}$ denote the set of $d_{\nu}$-tuples of functions $\varphi^{d}\left(1 \leq d \leq d_{\nu}\right)$ defined on $C$ and such that any pair $\left(\varphi^{a}, \varphi^{c}\right) \in \mathcal{V}_{\nu} \times \mathcal{V}_{\nu}$ is linked through the constraints (51) (with the index $b$ omitted).

Consequently, for any $\boldsymbol{x} \in C$ and any $s \in \mathcal{S}$, the value $\varphi(s \boldsymbol{x})$ of any vector function $\varphi \in \mathcal{U}_{\Sigma}$ at the image $s \boldsymbol{x}$ of $\boldsymbol{x}$ (i.e. at any point of $\Sigma$ ) can be decomposed by virtue of (49) and (50) into a weighted sum of the $n$ functions $\varphi_{\nu}^{c b}$ defined on the symmetry cell:

$$
\boldsymbol{\varphi}(s \boldsymbol{x})=\sum_{\mu=1}^{m} \sum_{b, c=1}^{d_{\mu}} \rho_{\mu}^{c b}\left(s^{-1}\right) s \boldsymbol{\varphi}_{\mu}^{c b}(\boldsymbol{x})
$$

Again, the basic plan is to find governing SGBEM formulations for the $n$ unknown functions $\varphi_{\nu}$, knowing that the solution to the original SGBEM equations can then be reconstructed at any point of $\Sigma$ by equation (30). 


\subsection{Exploitation of partial geometrical symmetry}

Again, the decomposition (31) holds. Inserting the decomposition (52) for both $\varphi$ and $\tilde{\varphi}$ in $\mathcal{B}\left(\varphi, \tilde{\varphi}^{\star}\right)$ defined by (31), one obtains:

$$
\begin{aligned}
\mathcal{B}\left(\boldsymbol{\varphi}, \tilde{\boldsymbol{\varphi}}^{\star}\right)=\sum_{\mu=1}^{m} \sum_{\nu=1}^{m} \sum_{b, c=1}^{d_{\mu}} \sum_{a, d=1}^{d_{\nu}} \sum_{s \in \mathcal{S}} \sum_{\tilde{s} \in \mathcal{S}} \rho_{\nu}^{d a \star}\left(\tilde{s}^{-1}\right) \rho_{\mu}^{c b}\left(s^{-1}\right) \\
\int_{C} \int_{C} B_{i k q s}(\tilde{s} \tilde{\boldsymbol{x}}, s \boldsymbol{x}) R_{s}\left[s \varphi_{\nu}^{c b}\right]_{k}(\boldsymbol{x}) R_{q}\left[\tilde{s} \tilde{\varphi}_{\mu}^{d a}\right]_{i}^{\star}(\tilde{\boldsymbol{x}}) \mathrm{d} S_{x} \mathrm{~d} S_{\tilde{x}}
\end{aligned}
$$

Then, making the change of variable $s=\tilde{s} t$ and using (44), (45):

$$
\begin{aligned}
\mathcal{B}\left(\boldsymbol{\varphi}, \tilde{\boldsymbol{\varphi}}^{\star}\right)= & \sum_{\mu=1}^{m} \sum_{\nu=1}^{m} \sum_{b, c=1}^{d_{\mu}} \sum_{d, a=1}^{d_{\nu}} \sum_{e=1}^{d_{\nu}} \sum_{t \in \mathcal{S}} \sum_{\tilde{s} \in \mathcal{S}} \\
& \rho_{\nu}^{a d}(\tilde{s}) \rho_{\mu}^{c e}\left(t^{-1}\right) \rho_{\mu}^{e b}\left(\tilde{s}^{-1}\right) \int_{C} \int_{C} B_{i k q s}(\tilde{s} \tilde{\boldsymbol{x}}, \tilde{s} t \boldsymbol{x}) R_{s}\left[\tilde{s} t \varphi_{\nu}^{c b}\right]_{k}(\boldsymbol{x}) R_{q}\left[\tilde{s} \tilde{\varphi}_{\mu}^{d a}\right]_{i}^{\star}(\tilde{\boldsymbol{x}}) \mathrm{d} S_{x} \mathrm{~d} S_{\tilde{x}}
\end{aligned}
$$

so that, using (46) and the equivariance property (21), one obtains:

$$
\begin{aligned}
\mathcal{B}\left(\boldsymbol{\varphi}, \tilde{\boldsymbol{\varphi}}^{\star}\right) & =\sum_{\mu=1}^{m} \sum_{\nu=1}^{m} \sum_{b, c=1}^{d_{\mu}} \sum_{d, a=1}^{d_{\nu}} \sum_{e=1}^{d_{\nu}} \sum_{t \in \mathcal{S}}\left\{\sum_{\tilde{s} \in \mathcal{S}} \rho_{\nu}^{a d}(\tilde{s}) \rho_{\mu}^{e b}\left(\tilde{s}^{-1}\right)\right\} \rho_{\mu}^{c e}\left(t^{-1}\right) \mathcal{B}_{t}\left(\boldsymbol{\varphi}_{\nu}^{c b}, \tilde{\boldsymbol{\varphi}}_{\mu}^{\star d a}\right) \\
& =\sum_{\mu=1}^{m} \sum_{\nu=1}^{m} \sum_{b, c=1}^{d_{\mu}} \sum_{d, a=1}^{d_{\nu}} \sum_{e=1}^{d_{\nu}} \sum_{t \in \mathcal{S}} \frac{n}{d_{\nu}} \rho_{\mu}^{c e}\left(t^{-1}\right) \delta_{a b} \delta_{d e} \delta_{\mu \nu} \mathcal{B}_{t}\left(\boldsymbol{\varphi}_{\nu}^{c b}, \tilde{\boldsymbol{\varphi}}_{\mu}^{\star d a}\right) \\
& =\sum_{\nu=1}^{m} \sum_{a=1}^{d_{\nu}} \sum_{c, d=1}^{d_{\nu}}\left\{\sum_{t \in \mathcal{S}} \frac{n}{d_{\nu}} \rho_{\nu}^{c d}\left(t^{-1}\right) \mathcal{B}_{t}\left(\boldsymbol{\varphi}_{\nu}^{c a}, \tilde{\boldsymbol{\varphi}}_{\nu}^{\star d a}\right)\right\} \\
& \equiv \sum_{\nu=1}^{m} \sum_{a=1}^{d_{\nu}} \sum_{c, d=1}^{d_{\nu}} \mathcal{B}_{\nu}^{c d}\left(\boldsymbol{\varphi}_{\nu}^{c a}, \tilde{\boldsymbol{\varphi}}_{\nu}^{\star d a}\right)
\end{aligned}
$$

where $\mathcal{B}_{t}(\cdot, \cdot)$ is again defined by $(36)$. One establishes in a similar way the decompositions:

$$
\mathcal{C}\left(\boldsymbol{\psi}, \tilde{\boldsymbol{\varphi}}^{\star}\right)=\sum_{\nu=1}^{m} \sum_{a, d=1}^{d_{\nu}} \mathcal{C}_{\nu}^{d a}\left(\boldsymbol{\psi}, \tilde{\boldsymbol{\varphi}}_{\nu}^{\star d a}\right) \quad \mathcal{F}\left(\tilde{\boldsymbol{\varphi}}^{\star}\right)=\sum_{\nu=1}^{m} \sum_{a, d=1}^{d_{\nu}} \mathcal{F}_{\nu}^{d a}\left(\tilde{\boldsymbol{\varphi}}_{\nu}^{\star d a}\right)
$$

with

$$
\begin{aligned}
\mathcal{C}_{\nu}^{d a}\left(\boldsymbol{\psi}, \tilde{\boldsymbol{\varphi}}_{\nu}^{\star d a}\right) & =\sum_{\tilde{s} \in \mathcal{S}} \rho_{\nu}^{a d}(\tilde{s}) \int_{C} \int_{\Gamma} B_{i k q s}(\tilde{s} \tilde{\boldsymbol{x}}, \boldsymbol{x}) R_{s} \psi_{k}(\boldsymbol{x}) R_{q}\left[\tilde{s} \tilde{\varphi}_{\nu}^{d a}\right]_{i}^{\star}(\tilde{\boldsymbol{x}}) \mathrm{d} S_{x} \mathrm{~d} S_{\tilde{x}} \\
\mathcal{F}_{\nu}^{d a}\left(\tilde{\boldsymbol{\varphi}}_{\nu}^{\star d a}\right) & =\frac{n}{d_{\nu}}\left\langle\overline{\boldsymbol{p}}_{\nu}^{d a}, \tilde{\boldsymbol{\varphi}}_{\nu}^{\star d a}\right\rangle_{C}
\end{aligned}
$$


Gathering results (53) and (54), the initial integral equation (6) reduces to a set of SGBIE problems of the form:

$$
\begin{aligned}
& \text { Find }\left\{\boldsymbol{\varphi}_{\nu}^{c a}\right\}_{1 \leq c \leq d_{\nu}} \in \mathcal{V}_{\nu}, \boldsymbol{\psi} \in \mathcal{U}_{\Gamma} ; \quad \forall\left\{\tilde{\boldsymbol{\varphi}}_{\nu}^{d}\right\}_{1 \leq d \leq d_{\nu}} \in \mathcal{V}_{\nu}, \boldsymbol{\psi} \in \mathcal{U}_{\Gamma} \\
& \left\{\begin{array}{c}
\sum_{c, d=1}^{d_{\nu}} \mathcal{B}_{\nu}^{c d}\left(\boldsymbol{\varphi}_{\nu}^{c a}, \tilde{\boldsymbol{\varphi}}_{\nu}^{\star d}\right)+\sum_{d=1}^{d_{\nu}} \mathcal{C}_{\nu}^{d a}\left(\boldsymbol{\psi}, \tilde{\boldsymbol{\varphi}}_{\nu}^{\star d}\right)=\sum_{d=1}^{d_{\nu}} \mathcal{F}_{\nu}^{d a}\left(\tilde{\boldsymbol{\varphi}}_{\nu}^{\star d}\right) \quad\left(1 \leq \nu \leq m, 1 \leq a \leq d_{\nu}\right) \\
\sum_{\nu=1}^{m} \sum_{a, d=1}^{d_{\nu}} \mathcal{C}_{\nu}^{d a}\left(\tilde{\boldsymbol{\psi}}^{\star}, \boldsymbol{\varphi}_{\nu}^{d a}\right)+\mathcal{D}\left(\boldsymbol{\psi}, \tilde{\boldsymbol{\psi}}^{\star}\right)=\mathcal{G}\left(\tilde{\boldsymbol{\psi}}^{\star}\right)
\end{array}\right.
\end{aligned}
$$

\subsection{Symmetry properties of the formulation (55).}

The symmetry properties of the operators $\mathcal{B}_{\nu}^{c d}(\boldsymbol{u}, \boldsymbol{v})$ associated with degree-one representations are as in the Abelian case, see section 4.3. Otherwise, one has from (53):

$$
\mathcal{B}_{\nu}^{c d}(\boldsymbol{u}, \boldsymbol{v})=\frac{n}{d_{\nu}} \sum_{t \in \mathcal{S}^{\prime}} \rho_{\nu}^{c d}\left(t^{-1}\right) \mathcal{B}_{t}(\boldsymbol{u}, \boldsymbol{v})+\frac{n}{d_{\nu}} \sum_{t \in \mathcal{S}^{\prime \prime}}\left\{\rho_{\nu}^{c d}(t) \mathcal{B}_{t}(\boldsymbol{v}, \boldsymbol{u})+\rho_{\nu}^{\star d c}(t) \mathcal{B}_{t}(\boldsymbol{u}, \boldsymbol{v})\right\}
$$

where $\mathcal{S}^{\prime}, \mathcal{S}^{\prime \prime} \subset \mathcal{S}$ are such that $\mathcal{S}^{\prime}=\left\{t \in \mathcal{S}, t=t^{-1}\right\}, \mathcal{S}^{\prime} \cap \mathcal{S}^{\prime \prime}=\emptyset$ and $\mathcal{S}=\mathcal{S}^{\prime} \cup \mathcal{S}^{\prime \prime} \cup\left\{t^{-1}, t \in \mathcal{S}^{\prime \prime}\right\}$. First, as a consequence of (40):

$$
\mathcal{B}_{t}(\boldsymbol{u}, \boldsymbol{v})=\mathcal{B}_{t^{-1}}(\boldsymbol{u}, \boldsymbol{v}) \quad\left(\text { if } t=t^{-1}\right)
$$

Also, whenever the irreducible representations $\rho_{\nu}^{c d}$ and $\rho_{\nu}^{d c}$ are real-valued, one has by virtue of (40) and (45) that

$$
\rho_{\nu}^{c d}(t) \mathcal{B}_{t}(\boldsymbol{v}, \boldsymbol{u})+\rho_{\nu}^{d c}(t) \mathcal{B}_{t}(\boldsymbol{u}, \boldsymbol{v})=\left[\rho_{\nu}^{d c}(t) \mathcal{B}_{t}(\boldsymbol{v}, \boldsymbol{u})+\rho_{\nu}^{c d}(t) \mathcal{B}_{t}(\boldsymbol{u}, \boldsymbol{v})\right]^{T}
$$

Besides, from (51):

$$
\left\{\boldsymbol{v}^{d}\right\}_{1 \leq d \leq d_{\nu}} \in \mathcal{V}_{\nu} \Rightarrow\left\{\boldsymbol{v}^{d \star}\right\}_{1 \leq d \leq d_{\nu}} \in \mathcal{V}_{\nu}
$$

Thus, if all $\rho_{\nu}^{c d}(t)$ are real-valued for a given $\nu$, the bilinear form

$$
\sum_{c, d=1}^{d_{\nu}} \mathcal{B}_{\nu}^{c d}\left(\boldsymbol{u}^{c}, \boldsymbol{v}^{\star d}\right)
$$

(where $\left\{\boldsymbol{u}^{c}\right\}_{1 \leq c \leq d_{\nu}} \in \mathcal{V}_{\nu}$ and $\left\{\boldsymbol{v}^{d}\right\}_{1 \leq d \leq d_{\nu}} \in \mathcal{V}_{\nu}$ ) is symmetric. On the other hand, if some $\rho_{\nu}^{c d}(t)$ are complex-valued, it is not clear how to establish the symmetry of the above bilinear form from the general properties of the representations.

Besides, it is also important to note that in (55) the same bilinear form $\sum_{c, d=1}^{d_{\nu}} \mathcal{B}_{\nu}^{c d}\left(\varphi^{c a}, \tilde{\varphi}^{\star d}\right)$ appears $d_{\nu}$ times; it should thus be assembled and factored once and then used to solve for all $d_{\nu^{-}}$uples $\left\{\boldsymbol{\varphi}_{\nu}^{c a}\right\}_{1 \leq c \leq d_{\nu}}$ with $a=1, \ldots, d_{\nu}$.

\section{AVOIDING RIGID-BODY EIGENSOLUTIONS}

It is well known that the three-dimensional interior elastostatic Neumann problem has eigensolutions of the form:

$$
\boldsymbol{U}(\boldsymbol{x})=\boldsymbol{a}+\boldsymbol{\omega} \cdot \boldsymbol{x} \quad \boldsymbol{x} \in \Omega
$$


where the constant vector $\boldsymbol{a}$ and the constant antisymmetric $3 \times 3$ matrix $\boldsymbol{\omega}$ are arbitrary. The set of all eigensolutions (58) is a six-dimensional vector space. In that case, one also has

$$
\mathcal{A}\left(\boldsymbol{U}, \tilde{\phi}^{\star}\right)=0 \quad \forall \tilde{\phi} \in \mathcal{U}
$$

for any field $\boldsymbol{U}$ of the form (58), where $\mathcal{A}\left(\boldsymbol{U}, \tilde{\boldsymbol{\phi}}^{\star}\right)$ is defined by (9).

More generally, let $\partial \Omega=\Sigma \cup \Gamma$ be such that $\Sigma=\Sigma_{1} \cup \ldots \cup \Sigma_{M} \cup \Sigma_{M+1} \cup \ldots \cup \Sigma_{M+M^{\prime}}$ and $\Gamma=$ $\Gamma_{1} \cup \ldots \cup \Gamma_{N} \cup \Gamma_{N+1} \cup \ldots \cup \Gamma_{N+N^{\prime}}$, where $\Omega_{\Sigma_{1}}, \ldots, \Omega_{\Sigma_{M}}$ and $\Omega_{\Gamma_{1}}, \ldots, \Omega_{\Gamma_{N}}$ have nonzero volume and $\Omega_{\Sigma_{M+1}}, \ldots, \Omega_{\Sigma_{M+M^{\prime}}}$ and $\Omega_{\Gamma_{N+1}}, \ldots, \Omega_{\Gamma_{N+N^{\prime}}}$ have zero volume (i.e., for practical purposes, the $\Sigma_{M+1}, \ldots, \Sigma_{M+M^{\prime}}$ and $\Gamma_{N+1}, \ldots, \Gamma_{N+N^{\prime}}$ define cracks). Then, any field $\boldsymbol{U}_{\mathrm{J}}(1 \leq J \leq M)$ such that

$$
\boldsymbol{U}_{\mathrm{J}}(\boldsymbol{x})=\boldsymbol{a}_{\mathrm{J}}+\boldsymbol{\omega}_{\mathrm{J}} \cdot \boldsymbol{x} \quad\left(\boldsymbol{x} \in \Sigma_{\mathrm{J}}\right) \quad \boldsymbol{U}_{\mathrm{J}}(\boldsymbol{x})=\mathbf{0} \quad\left(\boldsymbol{x} \in(\Sigma \cup \Gamma) \backslash \Sigma_{\mathrm{J}}\right)
$$

as well as any field $\boldsymbol{V}_{\mathrm{K}}(1 \leq K \leq N)$ such that

$$
\boldsymbol{V}_{\mathrm{K}}(\boldsymbol{x})=\boldsymbol{a}_{\mathrm{K}}+\boldsymbol{\omega}_{\mathrm{K}} \cdot \boldsymbol{x} \quad\left(\boldsymbol{x} \in \Gamma_{\mathrm{K}}\right) \quad \boldsymbol{V}_{\mathrm{K}}(\boldsymbol{x})=\mathbf{0} \quad\left(\boldsymbol{x} \in(\Sigma \cup \Gamma) \backslash \Gamma_{\mathrm{K}}\right)
$$

is an eigensolution of (6); in other words, the set of such eigensolutions defines a $6(M+N)$ dimensional vector subspace of $\mathcal{U}$ of the form $\mathcal{R}(\partial \Omega)=\mathcal{R}(\Sigma) \bigoplus \mathcal{R}(\Gamma)$, where $\mathcal{R}(\Sigma)$ and $\mathcal{R}(\Gamma)$ are generated by the eigensolutions (60) and (61), respectively.

One possible way to avoid these unwanted eigensolutions in (6) is to solve instead the modified SGBEM equation

$$
\mathcal{A}\left(\phi, \tilde{\phi}^{\star}\right)+\alpha \mathcal{E}\left(\phi, \tilde{\phi}^{\star}\right)=\mathcal{L}\left(\tilde{\phi}^{\star}\right) \quad(\forall \tilde{\phi} \in \mathcal{U})
$$

where $\mathcal{E}\left(\phi, \tilde{\phi}^{\star}\right)$ is a positive bilinear form of finite rank $6(M+N)$ such that

$$
\mathcal{E}\left(\boldsymbol{U}, \boldsymbol{U}^{\star}\right)>0 \quad(\forall \boldsymbol{U} \in \mathcal{R}(\partial \Omega), \boldsymbol{U} \neq \mathbf{0})
$$

Then, assuming that

$$
\mathcal{L}\left(\boldsymbol{U}^{\star}\right)=0 \quad(\forall \boldsymbol{U} \in \mathcal{R}(\partial \Omega))
$$

(which in particular is necessarily true for any interior Neumann problem when $M=1$ ), i.e. that each component $\Sigma_{\mathrm{J}}(1 \leq J \leq M)$ and $\Gamma_{\mathrm{K}}(1 \leq K \leq N)$ undergoes a self-equilibrated load, the solution $\hat{\phi}$ to the modified problem (62) is the unique solution to the original problem (6) which is orthogonal (in a $L^{2}$ sense) to $\mathcal{R}(\partial \Omega)$. One way to define $\mathcal{E}\left(\phi, \tilde{\phi}^{\star}\right)$ is:

$$
\begin{aligned}
\mathcal{E}\left(\boldsymbol{\phi}, \tilde{\boldsymbol{\phi}}^{\star}\right) & =\sum_{I=1}^{6} \sum_{J=1}^{M}\left\langle\boldsymbol{U}_{\mathrm{J}}^{\mathrm{I}}, \boldsymbol{\varphi}\right\rangle_{\Sigma}\left\langle\boldsymbol{U}_{\mathrm{J}}^{\mathrm{I}}, \tilde{\boldsymbol{\varphi}}^{\star}\right\rangle_{\Sigma}+\sum_{I=1}^{6} \sum_{K=1}^{N}\left\langle\boldsymbol{V}_{\mathrm{K}}^{\mathrm{I}}, \boldsymbol{\psi}\right\rangle_{\Gamma}\left\langle\boldsymbol{V}_{\mathrm{K}}^{\mathrm{I}}, \tilde{\boldsymbol{\psi}}^{\star}\right\rangle_{\Gamma} \\
& =\mathcal{E}_{\Sigma}\left(\boldsymbol{\varphi}, \tilde{\boldsymbol{\varphi}}^{\star}\right)+\mathcal{E}_{\Gamma}\left(\boldsymbol{\psi}, \tilde{\boldsymbol{\psi}}^{\star}\right)
\end{aligned}
$$

where each $\left\{\boldsymbol{U}_{\mathrm{J}}^{\mathrm{I}}, 1 \leq I \leq 6\right\}$ and $\left\{\boldsymbol{V}_{\mathrm{K}}^{\mathrm{I}}, 1 \leq I \leq 6\right\}$ is a set of 6 linearly independent vector functions of the form (60) and (61), respectively.

For the present purposes of exploiting symmetry, it turns out that $\mathcal{E}_{\Sigma}\left(\varphi, \tilde{\varphi}^{\star}\right)$ is not equivariant, i.e. does not fulfill the condition (15). It is thus better to use instead the modified form

$$
\mathcal{E}_{\Sigma}\left(\boldsymbol{\varphi}, \tilde{\boldsymbol{\varphi}}^{\star}\right)=\frac{1}{n} \sum_{s \in \mathcal{S}} \sum_{I=1}^{6} \sum_{K=1}^{M}\left\langle\mathcal{T}_{s} \boldsymbol{U}_{\mathrm{J}}^{\mathrm{I}}, \boldsymbol{\varphi}\right\rangle_{\Sigma}\left\langle\mathcal{T}_{s} \boldsymbol{U}_{\mathrm{J}}^{\mathrm{I}}, \tilde{\boldsymbol{\varphi}}^{\star}\right\rangle_{\Sigma}
$$

which reduces to the first contribution in the r.h.s. of (65) in the absence of geometrical symmetry (i.e. if $\mathcal{S}=\{I\}$ ) and is equivariant. Then, assuming $\partial \Omega$ is invariant under a (possibly non-Abelian) 
symmetry group $\mathcal{S}$ and decomposing $\phi, \tilde{\phi}$ and the $\boldsymbol{U}_{\mathrm{J}}^{\mathrm{I}}$ according to (30), one obtains after some algebraic manipulations:

$$
\mathcal{E}_{\Sigma}\left(\boldsymbol{\varphi}, \tilde{\boldsymbol{\varphi}}^{\star}\right)=\sum_{a=1}^{d_{\nu}} \sum_{\nu=1}^{m} \sum_{c, d=1}^{d_{\nu}} \mathcal{E}_{\nu}^{c d}\left(\boldsymbol{\varphi}_{\nu}^{c a}, \tilde{\boldsymbol{\varphi}}_{\nu}^{\star d a}\right)
$$

with

$$
\mathcal{E}_{\nu}^{c d}\left(\boldsymbol{\varphi}_{\nu}^{c a}, \tilde{\boldsymbol{\varphi}}_{\nu}^{\star d a}\right)=\sum_{b=1}^{d_{\nu}} \sum_{I=1}^{6} \sum_{K=1}^{M}\left\langle\left[\boldsymbol{U}_{\mathrm{J}}^{\mathrm{I}}\right]_{\nu}^{c b}, \boldsymbol{\varphi}_{\nu}^{c a}\right\rangle_{C}\left\langle\left[\boldsymbol{U}_{\mathrm{J}}^{\mathrm{I}}\right]_{\nu}^{d b}, \tilde{\boldsymbol{\varphi}}_{\nu}^{\star d a}\right\rangle_{C}
$$

The conclusion of this analysis is that, to avoid rigid-body eigensolutions in the symmetrydecomposed SGBEM formulations, one needs to replace $\mathcal{B}_{\nu}^{c d}\left(\varphi_{\nu}^{c a}, \tilde{\varphi}_{\nu}^{\star d}\right)$ by

$$
\mathcal{B}_{\nu}^{c d}\left(\boldsymbol{\varphi}_{\nu}^{c a}, \tilde{\varphi}_{\nu}^{\star d}\right)+\alpha \mathcal{E}_{\nu}^{c d}\left(\boldsymbol{\varphi}_{\nu}^{c a}, \tilde{\varphi}_{\nu}^{\star d}\right)
$$

(with $\alpha>0$ arbitrarily chosen), with $\mathcal{E}_{\nu}^{c d}\left(\varphi_{\nu}^{c a}, \tilde{\varphi}_{\nu}^{\star d}\right)$ defined by (68), in the subproblems (55).

\section{CALCULATION OF FIELD VALUES AT INTERIOR POINTS}

Displacement values at selected interior points $\tilde{\boldsymbol{x}}$ can be computed explicitly using the representation formula (2) once the density $\phi$ is known, and related quantities (strains, stresses) at $\tilde{\boldsymbol{x}}$ can be easily obtained as well.

Let $\boldsymbol{u}=\boldsymbol{v}+\boldsymbol{w}$ in (2), where $\boldsymbol{v}$ and $\boldsymbol{w}$ are the contributions of the integration over $\Sigma$ and $\Gamma$, respectively. Exploiting symmetry affects the computation of $\boldsymbol{v}$. Inserting the decomposition (52) into (2) and following the now usual pattern, one obtains:

$$
v_{k}(\tilde{s} \tilde{\boldsymbol{x}})=\sum_{s \in \mathcal{S}} \sum_{\nu=1}^{m} \sum_{a, b=1}^{d_{\nu}} \rho_{\nu}^{a b}\left(s^{-1}\right) \int_{C} T_{i}^{k}(\tilde{s} \tilde{\boldsymbol{x}}, s \boldsymbol{x}) s_{i j}\left[\varphi_{\nu}^{a b}\right]_{j}(\boldsymbol{x}) \mathrm{d} S_{x}
$$

Then, putting again $s=\tilde{s} t$ and using the equivariance property (21), which holds also for the kernel $T_{i}^{k}$, one obtains:

$$
v_{k}(\tilde{\boldsymbol{s}} \tilde{\boldsymbol{x}})=\sum_{t \in \mathcal{S}} \sum_{\nu=1}^{m} \sum_{a, b, c=1}^{d_{\nu}} \rho_{\nu}^{c b}\left(\tilde{s}^{-1}\right) s_{k \ell}\left\{\rho_{\nu}^{a c}\left(t^{-1}\right) \int_{C} T_{i}^{\ell}\left(t^{-1} \tilde{\boldsymbol{x}}, \boldsymbol{x}\right)\left[\varphi_{\nu}^{a b}\right]_{i}(\boldsymbol{x}) \mathrm{d} S_{x}\right\}
$$

(note that $s^{t} s=s s^{t}=I$ ). A close examination of (70) thus reveals that, for a given interior point $\boldsymbol{x}$, the same numerical quadrature effort is required by (2) and (70). However, the terms within curly brackets in (70) do not depend on $\tilde{s}$, so that the same numerical integrations can be reused (with different weights $\left.\rho_{\nu}^{c b}\left(\tilde{s}^{-1}\right)\right)$ to evaluate $\boldsymbol{v}$ at all the $n$ images of $\tilde{\boldsymbol{x}}$ under $\mathcal{S}$.

\section{IMPLEMENTATION ISSUES}

The symmetry cell $C$ and the surface $\Gamma$, as well as the unknown densities they support, are discretized in the usual boundary element fashion. For the Neumann problem used here as model situation, a continuous interpolation must be used for both $\phi$ and $\tilde{\phi}$. 


\subsection{Implementation of the boundary constraints}

The nodal values $\varphi_{\nu}^{c a}\left(\boldsymbol{x}^{q}\right)$ and $\tilde{\varphi}_{\nu}^{d}\left(\boldsymbol{x}^{q}\right)$ are unconstrained when $\boldsymbol{x}^{q}$ is an internal node of $C$. On the other hand, the boundary constraints (51) must be enforced at the nodes $\boldsymbol{x}^{q}$ located on the edge $\partial C$ of $C$; furthermore, these constraints hold for both $\phi$ and $\tilde{\phi}$. A general procedure for enforcing these constraints while maintaining the overall symmetry of the block-diagonalized set of discretized SGBEM equations, based on elimination and adapted from one proposed in [1], is now going to be presented.

Let $\boldsymbol{x}$ denote a generic point of $\partial C$, and let $s$ belong to the set $\mathcal{I}(\boldsymbol{x})$ defined by (28). Let $\left\{\boldsymbol{\varphi}_{\nu}(\boldsymbol{x})\right\}=\left\{\boldsymbol{\varphi}_{\nu}^{1}(\boldsymbol{x}) \ldots \boldsymbol{\varphi}_{\nu}^{d_{\nu}}(\boldsymbol{x})\right\}$ denote a $d_{\nu}$-uple of vectors attached to the point $\boldsymbol{x}$, arranged as a column vector of size $3 d_{\nu}$, and similarly for $\left\{\boldsymbol{\varphi}_{\nu}(s \boldsymbol{x})\right\}$. The constraint (51) on $\boldsymbol{\varphi}_{\nu}$ can be put in the form of a matrix equation as follows:

$$
[\boldsymbol{I}]\left\{\boldsymbol{\varphi}_{\nu}(s \boldsymbol{x})\right\}-\left[\rho_{\nu}(s) \otimes s\right]\left\{\boldsymbol{\varphi}_{\nu}(\boldsymbol{x})\right\}=\{\boldsymbol{0}\} \quad(\forall s \in \mathcal{I}(\boldsymbol{x}))
$$

where $[\boldsymbol{I}]$ denotes the identity matrix of size $\left(3 d_{\nu} \times 3 d_{\nu}\right)$ and $\otimes$ denotes the Kronecker product, i.e. $\left[\rho_{\nu}(s) \otimes s\right]$ is the $\left(3 d_{\nu} \times 3 d_{\nu}\right)$ block matrix:

$$
\left[\rho_{\nu}(s) \otimes s\right]=\left[\begin{array}{ccc}
\rho_{\nu}^{11}(s) s & \cdots & \rho_{\nu}^{1 d_{\nu}}(s) s \\
\vdots & & \vdots \\
\rho_{\nu}^{d_{\nu} 1}(s) s & \ldots & \rho_{\nu}^{d_{\nu} d_{\nu}}(s) s
\end{array}\right]
$$

Then, the treatment of the boundary constraints (51) will depend on whether $s \boldsymbol{x}=\boldsymbol{x}$ or $s \boldsymbol{x} \neq \boldsymbol{x}$. Accordingly, introduce the following splitting of $\mathcal{I}(\boldsymbol{x})$ :

$$
\mathcal{I}(\boldsymbol{x})=\mathcal{I}_{0}(\boldsymbol{x}) \cup \mathcal{I}_{1}(\boldsymbol{x}) \quad \text { with } \mathcal{I}_{0}(\boldsymbol{x})=\{s \in \mathcal{I}(\boldsymbol{x}), s \boldsymbol{x}=\boldsymbol{x}\} \text { and } \mathcal{I}_{1}(\boldsymbol{x})=\mathcal{I}(\boldsymbol{x}) \backslash \mathcal{I}_{0}(\boldsymbol{x})
$$

where $\mathcal{I}_{0}(\boldsymbol{x})$ thus denotes the set of isometries under which $\boldsymbol{x} \in \partial C$ is a fixed point. It is not difficult to show that $\mathcal{I}_{0}(\boldsymbol{x})$ is, for any $\boldsymbol{x} \in \partial C$, a subgroup of $\mathcal{S}$ (possibly reduced to $\mathcal{I}(\boldsymbol{x})=\{I\}$ ).

If $s \in \mathcal{I}_{1}(\boldsymbol{x})$ (this possibility may occur e.g. in symmetry groups which involve cyclic symmetry), then equation (71) gives $\left\{\boldsymbol{\varphi}_{\nu}(s \boldsymbol{x})\right\}$ explicitly in terms of $\left\{\boldsymbol{\varphi}_{\nu}(\boldsymbol{x})\right\}$, thus allowing for a straightforward elimination of $\left\{\boldsymbol{\varphi}_{\nu}(s \boldsymbol{x})\right\}$ (i.e. performed columnwise). The same elimination must be performed with respect to $\left\{\tilde{\boldsymbol{\varphi}}_{\nu}(s \boldsymbol{x})\right\}$, i.e. row-wise, to preserve algebraic symmetry.

If $s \in \mathcal{I}_{0}(\boldsymbol{x})$, then equation (71) becomes a set of homogeneous matrix equations of size $\left(3 d_{\nu}\right) \times\left(3 d_{\nu}\right):$

$$
\left([\boldsymbol{I}]-\left[\rho_{\nu}(s) \otimes s\right]\right)\left\{\boldsymbol{\varphi}_{\nu}(\boldsymbol{x})\right\}=\{\boldsymbol{0}\} \quad\left(\forall s \in \mathcal{I}_{0}(\boldsymbol{x})\right)
$$

Note that, in the special case where $\mathcal{I}_{0}(\boldsymbol{x})=\{I\},\left[\rho_{\nu}(s) \otimes s\right]=[\boldsymbol{I}]$ and $\left\{\boldsymbol{\varphi}_{\nu}(\boldsymbol{x})\right\}$ is unconstrained, as expected. Now, let

$$
[\boldsymbol{R}]=\sum_{s \in \mathcal{I}_{0}(\boldsymbol{x})}\left([\boldsymbol{I}]-\left[\rho_{\nu}(s) \otimes s\right]\right)
$$

Obviously, (74) implies that $[\boldsymbol{R}]\left\{\boldsymbol{\varphi}_{\nu}(\boldsymbol{x})\right\}=\{\boldsymbol{0}\}$. Conversely, one can check by direct inspection the identity

$$
\left([\boldsymbol{I}]-\left[\rho_{\nu}(s) \otimes s\right]\right)[\boldsymbol{R}]=[\boldsymbol{I}]-\left[\rho_{\nu}(s) \otimes s\right] \quad\left(\forall s \in \mathcal{I}_{0}(\boldsymbol{x})\right)
$$

which implies that

$$
[\boldsymbol{R}]\left\{\boldsymbol{\varphi}_{\nu}(\boldsymbol{x})\right\}=\{\boldsymbol{0}\} \quad \Longrightarrow \quad\left([\boldsymbol{I}]-\left[\rho_{\nu}(s) \otimes s\right]\right)\left\{\boldsymbol{\varphi}_{\nu}(\boldsymbol{x})\right\}=\{\mathbf{0}\}
$$


Thus, equation (74) is equivalent to the single matrix equation

$$
[\boldsymbol{R}]\left\{\boldsymbol{\varphi}_{\nu}(\boldsymbol{x})\right\}=\{\mathbf{0}\}
$$

Besides, it can be shown that $[\boldsymbol{R}]$ is symmetric and that

$$
[\boldsymbol{R}][\boldsymbol{R}]=[\boldsymbol{R}]
$$

i.e. that $[\boldsymbol{R}]$ defines a projection in a $3 d_{\nu}$-dimensional vector space. Thus, $[\boldsymbol{R}]$ admits an eigenvalue decomposition of the form

$$
[\boldsymbol{R}]=\sum_{i=1}^{3 d_{\nu}} \lambda_{i}\left\{\boldsymbol{a}_{i}\right\} \otimes\left\{\boldsymbol{a}_{i}\right\}
$$

where the $\left\{\boldsymbol{a}_{i}\right\}$ are orthonormal $3 d_{\nu}$-vectors and $(\forall i) \lambda_{i}=1$ or 0 . Hence, any $\left\{\boldsymbol{\varphi}_{\nu}(\boldsymbol{x})\right\}$ solving (74) has the form

$$
\left\{\boldsymbol{\varphi}_{\nu}(\boldsymbol{x})\right\}=\sum_{i \in J_{0}(\boldsymbol{x})}\left\{\boldsymbol{a}_{i}\right\} y_{i}
$$

where $J_{0}(\boldsymbol{x})=\left\{i, \lambda_{i}=1\right\}$ and the $y_{i}\left(i \in J_{0}(\boldsymbol{x})\right)$ are arbitrary. Any $\left\{\boldsymbol{\varphi}_{\nu}(\boldsymbol{x})\right\}$ solving (74) is thus expressed in terms of a new set of unconstrained unknowns $y_{i}\left(i \in J_{0}(\boldsymbol{x})\right)$, of size smaller that $3 d_{\nu}$. Similarly, $\left\{\tilde{\boldsymbol{\varphi}}_{\nu}(\boldsymbol{x})\right\}$ solving (74) is expressed in terms of a new, smaller, trial vector $\tilde{y}_{i}\left(i \in J_{0}(\boldsymbol{x})\right)$. Performing these substitutions into the block-diagonal part of the SGBEM system of equations allows to recast each block as a slightly smaller, symmetric block acting on unconstrained unknowns.

\subsection{Reduction of numerical quadrature effort}

Without exploiting symmetry, the numerical quadrature entails a computing time $T_{I, N}$, of which an estimate is $T_{I, N}^{e}=O\left(\left(n M_{1}+M_{2}\right)^{2}\right)$, where $M_{1}$ and $M_{2}$ are the numbers of boundary elements supported by $C$ and $\Gamma$, respectively. It is obvious from (79) that a reduction of both setup and solution computational efforts results from the block-diagonalization of the operator $\mathcal{B}$. The numerical quadrature effort consists in evaluating discretized versions of

$$
\mathcal{B}_{t}(\boldsymbol{u}, \boldsymbol{v}) \equiv \int_{C} \int_{C} B(t \boldsymbol{x}, \tilde{\boldsymbol{x}} ; \boldsymbol{t} \boldsymbol{u}, \boldsymbol{v}) \mathrm{d} S_{x} \mathrm{~d} S_{\tilde{x}}
$$

for all $t \in \mathcal{S}$ instead of

$$
\mathcal{B}(\boldsymbol{u}, \boldsymbol{v}) \equiv \int_{\Sigma} \int_{\Sigma} B(\boldsymbol{x}, \tilde{\boldsymbol{x}} ; \boldsymbol{u}, \boldsymbol{v}) \mathrm{d} S_{x} \mathrm{~d} S_{\tilde{x}}
$$

From this identity and the symmetry of the original bilinear form $\mathcal{B}$, the block-diagonalized $\mathcal{B}$ is seen (assuming for simplicity that the same quadrature rule is used for all integrations) to entail a numerical quadrature effort $n$ times smaller than the original $\mathcal{B}$. In the case of partial symmetry, an estimation of the time $T_{I, S}$ needed for performing all numerical integrations is $T_{I, S}^{e}=O\left(n M_{1}^{2}+2 n M_{1} M_{2}+M_{2}^{2}\right)$. Letting

$$
\tau_{I}^{e}=\frac{T_{I, S}^{e}}{T_{I, N}^{e}}=\frac{n M_{1}^{2}+2 n M_{1} M_{2}+M_{2}^{2}}{\left(n M_{1}+M_{2}\right)^{2}}
$$

denote the expected ratio of integration CPU times with and without exploitation of (partial) symmetry, we have as expected that $\tau_{I}^{e}=1 / n$ if $M_{2}=0$ (i.e. complete symmetry). 


\subsection{Reduction in solution time}

Let $n N_{1}$ and $N_{2}$ denote the number of degrees of freedom (DOFs) supported by the BEM discretization of $\Sigma$ and $\Gamma$ respectively, so that $N_{1}$ is the average number of DOFs supported by the symmetry cell $C$ (the treatment proposed in Section 8.1 for the constraints (51) imply that the various subproblems do not have exactly the same size even for a given value of the degree $d_{\nu}$, as exemplified by Table VIII for the examples of section 10). The system of equations (39) or (55) takes the general form:

$$
\left[\begin{array}{cc}
\boldsymbol{B} & C \\
\boldsymbol{C}^{T} & \boldsymbol{D}
\end{array}\right]\left\{\begin{array}{l}
\boldsymbol{\varphi} \\
\boldsymbol{\psi}
\end{array}\right\}=\left[\begin{array}{l}
\boldsymbol{F} \\
\boldsymbol{G}
\end{array}\right]
$$

where the matrix $\boldsymbol{B}$ is block-diagonal: $\boldsymbol{B}=\operatorname{Diag}\left(\boldsymbol{B}_{\nu}^{a}\right)\left(1 \leq \nu \leq m, 1 \leq a \leq d_{\nu}\right)$. Each block $\boldsymbol{B}_{\nu}^{a}$ is approximately of size $d_{\nu} \times N_{1}$. Besides, as mentioned before, all blocks $\bar{B}_{\nu}^{a}\left(1 \leq a \leq d_{\nu}\right)$ are the same for a given $\nu$. For example, the developed form of (79) for the dihedral group $\bar{D}^{3}$ (see Appendix II) is:

$$
\left[\begin{array}{ccccccc}
\boldsymbol{B}_{1} & \mathbf{0} & \mathbf{0} & \mathbf{0} & \mathbf{0} & \mathbf{0} & \boldsymbol{C}_{1} \\
\mathbf{0} & \boldsymbol{B}_{2} & \mathbf{0} & \mathbf{0} & \mathbf{0} & \mathbf{0} & \boldsymbol{C}_{2} \\
\mathbf{0} & \mathbf{0} & \boldsymbol{B}_{3}^{11} & \boldsymbol{B}_{3}^{21} & \mathbf{0} & \mathbf{0} & \boldsymbol{C}_{3}^{11} \\
\mathbf{0} & \mathbf{0} & \boldsymbol{B}_{3}^{12} & \boldsymbol{B}_{3}^{22} & \mathbf{0} & \mathbf{0} & \boldsymbol{C}_{3}^{21} \\
\mathbf{0} & \mathbf{0} & \mathbf{0} & \mathbf{0} & \boldsymbol{B}_{3}^{11} & \boldsymbol{B}_{3}^{21} & \boldsymbol{C}_{3}^{12} \\
\mathbf{0} & \mathbf{0} & \mathbf{0} & \mathbf{0} & \boldsymbol{B}_{3}^{12} & \boldsymbol{B}_{3}^{22} & \boldsymbol{C}_{3}^{22} \\
\boldsymbol{C}_{1}^{T} & \boldsymbol{C}_{2}^{T} & \boldsymbol{C}_{3}^{11 T} & \boldsymbol{C}_{3}^{21 T} & \boldsymbol{C}_{3}^{12 T} & \boldsymbol{C}_{3}^{22 T} & \boldsymbol{D}
\end{array}\right]\left\{\begin{array}{c}
\boldsymbol{\varphi}_{1} \\
\boldsymbol{\varphi}_{2} \\
\boldsymbol{\varphi}_{3}^{11} \\
\varphi_{3}^{21} \\
\boldsymbol{\varphi}_{3}^{12} \\
\varphi_{3}^{22} \\
\boldsymbol{\psi}
\end{array}\right\}=\left\{\begin{array}{c}
\boldsymbol{F}_{1} \\
\boldsymbol{F}_{2} \\
\boldsymbol{F}_{3}^{11} \\
\boldsymbol{F}_{3}^{21} \\
\boldsymbol{F}_{3}^{12} \\
\boldsymbol{F}_{3}^{22} \\
\boldsymbol{G}
\end{array}\right\}
$$

Solving the original (symmetric) SGBEM system thus entails a solution time $T_{R, N}^{e}$ for which an estimate is $T_{R, N}^{e}=O\left(\left(n N_{1}+N_{2}\right)^{3} / 6\right)$. For solving the system (79), the following steps are to be performed, with the corresponding computation times $T_{R, S}^{(i)}$ as indicated:

1. Blockwise Choleski factorization of $\boldsymbol{B}=\operatorname{Diag}\left(\boldsymbol{B}_{\nu}^{a}\right)$ so that $\left[\boldsymbol{B}_{\nu}^{a}\right]=\left[\boldsymbol{R}_{\nu}^{a}\right]^{\mathrm{T}}\left[\boldsymbol{R}_{\nu}^{a}\right]$ :

$$
T_{R, S}^{(1)}=\frac{N_{1}^{3}}{6} \sum_{\nu=1}^{m} d_{\nu}^{3}+\mathcal{P}_{2}\left(N_{1}, N_{2}\right)
$$

2. Solution of the systems $\left[\boldsymbol{R}_{\nu}^{a}\right]^{\mathrm{T}}\left\{\boldsymbol{z}_{\nu}^{a}\right\}=\left\{\boldsymbol{F}_{\nu}^{a}\right\}$ and $\left[\boldsymbol{R}_{\nu}^{a}\right]^{\mathrm{T}}\left[\boldsymbol{Z}_{\nu}^{a}\right]=\left[\boldsymbol{C}_{\nu}^{a}\right]$ :

$$
T_{R, S}^{(2)}=\frac{N_{1}^{2} N_{2}}{2} \sum_{\nu=1}^{m} d_{\nu}^{3}+\mathcal{P}_{2}\left(N_{1}, N_{2}\right)
$$

3. Computation of $[\hat{\boldsymbol{D}}]=[\boldsymbol{D}]-\sum_{\nu=1}^{m} \sum_{a=1}^{d_{\nu}}\left[\boldsymbol{Z}_{\nu}^{a}\right]^{\mathrm{T}}\left[\boldsymbol{Z}_{\nu}^{a}\right]$ and $\{\hat{\boldsymbol{G}}\}=\{\boldsymbol{G}\}-\sum_{\nu=1}^{m} \sum_{a=1}^{d_{\nu}}\left[\boldsymbol{Z}_{\nu}^{a}\right]^{\mathrm{T}}\left[\boldsymbol{z}_{\nu}^{a}\right]$ :

$$
T_{R, S}^{(3)}=\frac{n N_{1} N_{2}^{2}}{2}+\mathcal{P}_{2}\left(N_{1}, N_{2}\right)
$$

4. Factorization and solution of $[\hat{D}]\{\boldsymbol{\psi}\}=\{\hat{\boldsymbol{G}}\}$ :

$$
T_{R, S}^{(4)}=\frac{N_{2}^{3}}{6}+\mathcal{P}_{2}\left(N_{1}, N_{2}\right)
$$

5. Solution of the systems $\left[\boldsymbol{R}_{\nu}^{a}\right]\left\{\boldsymbol{\varphi}_{\nu}^{a}\right\}=\left\{\boldsymbol{z}_{\nu}^{a}\right\}-\left[\boldsymbol{Z}_{\nu}^{a}\right]\{\boldsymbol{\psi}\}$ :

$$
T_{R, S}^{(5)}=\mathcal{P}_{2}\left(N_{1}, N_{2}\right)
$$


where the generic notation $\mathcal{P}_{2}\left(N_{1}, N_{2}\right)$ indicates an unspecified polynomial of total degree 2 in $N_{1}, N_{2}$. Thus, the estimate $T_{R, S}^{e}$ of the CPU time $T_{R, S}$ needed for solving (79) (retaining only the $O\left(N^{3}\right)$ contributions) is

$$
\begin{aligned}
T_{R, S}^{e}=T_{R, S}^{(1)}+T_{R, S}^{(2)}+T_{R, S}^{(3)} & +T_{R, S}^{(4)}+\mathcal{P}_{2}\left(N_{1}, N_{2}\right) \\
& =\frac{1}{6}\left[N_{2}^{3}+3 n N_{2}^{2} N_{1}+\left(3 N_{2} N_{1}^{2}+N_{1}^{3}\right) \sum_{\nu=1}^{m} d_{\nu}^{3}\right]+\mathcal{P}_{2}\left(N_{1}, N_{2}\right)
\end{aligned}
$$

Let $\tau_{R}^{e}$ denote the expected ratio of solution CPU times with and without exploitation of (partial) symmetry, i.e.:

$$
\tau_{R}^{e}=\frac{T_{R, S}^{e}}{T_{R, N}^{e}}=\frac{N_{2}^{3}+3 n N_{2}^{2} N_{1}+\left(3 N_{2} N_{1}^{2}+N_{1}^{3}\right) \sum_{\nu=1}^{m} d_{\nu}^{3}}{\left(n N_{1}+N_{2}\right)^{3}}
$$

For instance, with $N_{2}=0$ (i.e. complete symmetry), one has $\tau_{R}^{e}=\left(1 / n^{3}\right) \sum_{\nu=1}^{R(n)} d_{\nu}^{3}$. Obviously, the highest gains in solution time occur for $n$ large (i.e. high degrees of symmetry) and $N_{2} /\left(n N_{1}\right)$ small. Also, $\sum_{\nu=1}^{m} d_{\nu}^{3}=n$ if $\mathcal{S}$ is Abelian, hence in that case $\tau_{R}^{e}=1 / n^{2}$ with $N_{2}=0$ as expected.

Steps 1 and 2 in the foregoing analysis rest on the assumption that all blocks $\left[\boldsymbol{B}_{\nu}^{a}\right]$ are symmetric. The case where reciprocal symmetry occurs, i.e. $\left[\boldsymbol{B}_{\nu}^{a}\right]$ not symmetric but $\left[\boldsymbol{B}_{\nu^{\star}}^{a}\right]=\left[\boldsymbol{B}_{\nu}^{a}\right]^{\mathrm{T}}$ (see end of section 4), can be analysed in a similar fashion, and the estimate (80) still holds.

\subsection{Complex representations and real-valued problems}

The model problem (1) is real-valued, as is the kernel function $B_{i k q s}$. However, Abelian symmetry groups in the physical space $\mathbb{R}^{3}$ often have complex-valued representations, in which case Eqs. (25), (37), (38) show that the subproblems (39) are in general complex-valued. In fact, it is easy to show in this case that:

$$
\mathcal{B}_{\nu^{\star}}(\varphi, \tilde{\varphi})=\left[\mathcal{B}_{\nu}\right]^{\star}(\varphi, \tilde{\varphi}) \quad \mathcal{C}_{\nu^{\star}}(\varphi, \tilde{\varphi})=\left[\mathcal{C}_{\nu}\right]^{\star}(\boldsymbol{\varphi}, \tilde{\varphi}) \quad \mathcal{F}_{\nu^{\star}}(\tilde{\varphi})=\left[\mathcal{F}_{\nu}\right]^{\star}(\tilde{\varphi})
$$

Thus, the equations for the $\nu$-subproblem and the $\nu^{\star}$-subproblem, and hence their solutions $\left(\boldsymbol{\varphi}_{\nu}, \boldsymbol{\varphi}_{\nu^{\star}}\right)$, are conjugate to each other and thus redundant. It is sufficient to solve (say) the $\nu$-subproblem for $\varphi_{\nu}$. The contribution of the conjugate pair $\left(\varphi_{\nu}, \varphi_{\nu^{\star}}\right)$ to the reconstruction of the (real) global solution $u$ is then:

$$
\left[\boldsymbol{P}_{\nu} \boldsymbol{\varphi}\right](s \boldsymbol{x})+\left[\boldsymbol{P}_{\nu^{\star}} \boldsymbol{\varphi}\right](s \boldsymbol{x})=\rho_{\nu}^{\star}(s) \boldsymbol{\varphi}_{\nu}+\rho_{\nu}(s) \boldsymbol{\varphi}_{\nu}^{\star}=2 \operatorname{Re}\left(\rho_{\nu}^{\star}(s) \boldsymbol{\varphi}_{\nu}\right)
$$

In the FEM framework, adequate combinations of the two conjugate equations are known to yield two coupled real-valued subproblems defined on the (volumic) symmetry cell. Here, a similar approach could be applied to the symmetry-reduced SGBEM. However, contrarily to the FEM case, this would result in one subproblem of size $2 N / n$, and hence would not bring any advantage over solving directly the complex-valued subproblem of size $N / n$.

\section{EXTENSION TO SCALAR, COMPLEX-VALUED OR DIRICHLET PROBLEMS}

The methodology of sections 4 and 5 can be applied in exactly the same way to linear complex-valued scalar or vector boundary-value problems (e.g. linear acoustics, elastodynamics or electromagnetism in the frequency domain), for which the fundamental solutions are complex-valued. It is useful to 
recall that the integral operators, e.g. the elastodynamic counterpart of $\mathcal{A}$ in (6), is symmetric but not Hermitian, i.e. $\mathcal{A}\left(\phi, \tilde{\phi}^{\star}\right)=\mathcal{A}\left(\tilde{\phi}^{\star}, \phi\right)$; in particular, the discussion of sections 4.3 and 5.3 is still relevant. Also, the case of Dirichlet boundary conditions does not raise any further difficulties. For example, the methodology of sections 4 and 5 is directly applicable to the Dirichlet elastostatic BVP, starting from the governing SGBEM formulation associated with representing the displacement field in terms of a single-layer elastic potential

$$
u_{k}(\tilde{\boldsymbol{x}})=\int_{\partial \Omega} U_{i}^{k}(\tilde{\boldsymbol{x}}, \boldsymbol{x}) \phi_{i}(\boldsymbol{x}) \mathrm{d} S_{x}
$$

instead of the double-layer potential (2) used for the Neumann BVP. In particular, the strategy developed in section 6 to circumvent rigid-body type eigensolutions is clearly relevant only for Neumann elastostatic problems.

To exemplify the foregoing discussion, and for the sake of completeness, we now present the initial and symmetry-reduced SGBEM formulations for the Helmholtz equation with Dirichlet boundary conditions, where a scalar complex-valued unknown function $u$ is governed by:

$$
\left.\left(\Delta+k^{2}\right) u=0 \quad \text { (in } \Omega\right) \quad u=\bar{u} \quad(\text { on } \partial \Omega)
$$

where $k$ is the wavenumber. The indirect SGBEM formulation for problem (83) is:

$$
\text { Find } \phi \in \mathcal{U}=H^{-1 / 2}(\partial \Omega), \quad \mathcal{A}\left(\phi, \tilde{\phi}^{\star}\right)=\mathcal{L}\left(\tilde{\phi}^{\star}\right) \quad(\forall \tilde{\phi} \in \mathcal{U})
$$

with

$$
\mathcal{A}\left(\phi, \tilde{\phi}^{\star}\right)=\int_{\partial \Omega} \int_{\partial \Omega} G(\tilde{\boldsymbol{x}}, \boldsymbol{x}) \phi(\boldsymbol{x}) \tilde{\phi}^{\star}(\tilde{\boldsymbol{x}}) \mathrm{d} S_{x} \mathrm{~d} S_{\tilde{x}} \quad \mathcal{L}\left(\tilde{\phi}^{\star}\right)=\left\langle\bar{u}, \tilde{\phi}^{\star}\right\rangle_{\partial \Omega}
$$

where $G(\tilde{\boldsymbol{x}}, \boldsymbol{x})=\exp (i k r) /(4 \pi r)$ is the well-known full-space fundamental solution of the Helmholtz equation, which has the symmetry property $G(\tilde{\boldsymbol{x}}, \boldsymbol{x})=G(\boldsymbol{x}, \tilde{\boldsymbol{x}})$.

Then, exploitation of partial geometrical symmetry follows the steps of section 5. It is however necessary to adapt some of the definitions of section 5.1 to the fact that the unknown and trial functions are now scalar. In particular, the projectors $P_{\nu}^{a b}$ are now defined by

$$
\left[P_{\nu}^{a b} v\right](\boldsymbol{x})=\frac{d_{\nu}}{n} \sum_{t \in \mathcal{S}} \rho_{\nu}^{\star a b}(t) v\left(t^{-1} \boldsymbol{x}\right)
$$

instead of (48), the counterpart of the reconstruction formula (52) is

$$
v(s \boldsymbol{x})=\sum_{\mu=1}^{m} \sum_{b, c=1}^{d_{\mu}} \rho_{\mu}^{c b}\left(s^{-1}\right) v_{\mu}^{c b}(\boldsymbol{x})
$$

and the constraints to be satisfied on $\partial C$ are

$$
v_{\nu}^{a b}(s \boldsymbol{x})-\sum_{c=1}^{d_{\nu}} \rho_{\nu}^{c a}\left(s^{-1}\right) v_{\nu}^{c b}(\boldsymbol{x})=0 \quad(\forall \boldsymbol{x} \in \partial C, \forall s \in \mathcal{I}(\boldsymbol{x}))
$$

instead of (51). The following set of SGBIE problems is thus obtained (with $\mathcal{U}_{\Gamma}=H^{-1 / 2}(\Gamma)$ ):

$$
\begin{aligned}
& \text { Find }\left\{\varphi_{\nu}^{c a}\right\}_{1 \leq c \leq d_{\nu}} \in \mathcal{V}_{\nu}, \psi \in \mathcal{U}_{\Gamma} ; \quad \forall\left\{\tilde{\varphi}_{\nu}^{d}\right\}_{1 \leq d \leq d_{\nu}} \in \mathcal{V}_{\nu}, \psi \in \mathcal{U}_{\Gamma} \\
& \qquad\left\{\begin{array}{c}
\sum_{c, d=1}^{d_{\nu}} \mathcal{B}_{\nu}^{c d}\left(\varphi_{\nu}^{c a}, \tilde{\varphi}_{\nu}^{\star d}\right)+\sum_{d=1}^{d_{\nu}} \mathcal{C}_{\nu}^{d a}\left(\psi, \tilde{\varphi}_{\nu}^{\star d}\right)=\sum_{d=1}^{d_{\nu}} \mathcal{F}_{\nu}^{d a}\left(\tilde{\varphi}_{\nu}^{\star d}\right) \quad\left(1 \leq \nu \leq m, 1 \leq a \leq d_{\nu}\right) \\
\sum_{\nu=1}^{m} \sum_{a, d=1}^{d_{\nu}} \mathcal{C}_{\nu}^{d a}\left(\tilde{\psi}^{\star}, \varphi_{\nu}^{d a}\right)+\mathcal{D}\left(\psi, \tilde{\psi}^{\star}\right)=\mathcal{G}\left(\tilde{\psi}^{\star}\right)
\end{array}\right.
\end{aligned}
$$


with

$$
\begin{aligned}
\mathcal{B}_{\nu}^{c d}\left(\varphi_{\nu}^{c a}, \tilde{\varphi}_{\nu}^{\star d}\right) & =\sum_{t \in \mathcal{S}} \frac{n}{d_{\nu}} \rho_{\nu}^{c d}\left(t^{-1}\right) \int_{C} \int_{C} G(\tilde{\boldsymbol{x}}, t \boldsymbol{x}) \varphi_{\nu}^{c a}(\boldsymbol{x}) \tilde{\varphi}_{\nu}^{\star d}(\tilde{\boldsymbol{x}}) \mathrm{d} S_{x} \mathrm{~d} S_{\tilde{x}} \\
\mathcal{C}_{\nu}^{d a}\left(\psi, \tilde{\varphi}_{\nu}^{\star d}\right) & =\sum_{\tilde{s} \in \mathcal{S}} \rho_{\nu}^{a d}(\tilde{s}) \int_{C} \int_{\Gamma} G(\tilde{s} \tilde{\boldsymbol{x}}, \boldsymbol{x}) \psi(\boldsymbol{x}) \tilde{\varphi}_{\nu}^{\star d}(\tilde{\boldsymbol{x}}) \mathrm{d} S_{x} \mathrm{~d} S_{\tilde{x}} \\
\mathcal{F}_{\nu}^{d a}\left(\tilde{\varphi}_{\nu}^{\star d}\right) & =\frac{n}{d_{\nu}}\left\langle\overline{\boldsymbol{p}}_{\nu}^{d a}, \tilde{\varphi}_{\nu}^{\star d}\right\rangle_{C} \\
\mathcal{D}\left(\psi, \tilde{\psi}^{\star}\right) & =\int_{\Gamma} \int_{\Gamma} G(\tilde{\boldsymbol{x}}, \boldsymbol{x}) \psi(\boldsymbol{x}) \tilde{\psi}^{\star}(\tilde{\boldsymbol{x}}) \mathrm{d} S_{x} \mathrm{~d} S_{\tilde{x}} \\
\mathcal{G}\left(\tilde{\psi}^{\star}\right) & =\left\langle\bar{u}, \tilde{\psi}^{\star}\right\rangle_{\Gamma}
\end{aligned}
$$

where $\varphi, \tilde{\varphi} \in \mathcal{U}_{\Sigma}=\left[H^{-1 / 2}(\Sigma)\right]^{3}$ and $\psi, \tilde{\psi} \in \mathcal{U}_{\Gamma}$ denote the restrictions of $\phi, \tilde{\phi}$ on $\Sigma$ and on $\Gamma$.

\section{NUMERICAL IMPLEMENTATION AND EXAMPLES}

The concepts developed in the previous sections have been implemented using the MATLAB environment. This choice was primarily motivated by convenience, MATLAB being well suited to programming within limited time resources. Eight-noded curved quadrilateral boundary elements are used for both geometrical and physical interpolations. Special quadrature algorithms for all types of 4-dimensional weakly singular $|\boldsymbol{x}-\tilde{\boldsymbol{x}}|^{-1}$ double element integrals (coincident, edge-adjacent, vertex-adjacent) have been used. The disjoint (i.e. nonsingular) double element integrals are evaluated using quadrature rules for nonsingular integrations over 4-cubes [10]. Direct Choleski-based solvers (invoking the built-in Chol operator of MATLAB) have been used, in connection with the solution algorithm described in section 8.3 when partial symmetry is exploited. In some cases (as indicated below), a conjugate-gradient iterative solver has been used as well.

Numerical examples are now presented in order to demonstrate and assess the computational efficiency of the proposed method. For each example, a table displays (using the notations defined in sections 8.2 and 8.3) the relevant mesh parameters $M_{1}, M_{2}, N_{1}, N_{2}$ together with the recorded CPU times $T_{I, S}, T_{I, N}, T_{R, S}, T_{R, N}$ used for numerical integration and solution, with and without symmetry (note that $M_{2}, N_{2}$ are relevant only in cases of partial symmetry). The ratios $\tau_{I} / \tau_{I}^{e}$ and $\tau_{R} / \tau_{R}^{e}$, which compare the actual gains $\tau_{I}, \tau_{R}$ in CPU time to their theoretical estimates $\tau_{I}^{e}, \tau_{R}^{e}$ defined by (78) and (81), are also presented. The elastic moduli are $E=1 \mathrm{MPa}$ and $\nu=0.3$ for all examples. All computations have been performed on a Linux PC with one Pentium III, $700 \mathrm{MHz}$ processor.

\subsection{Example 1: pressurized spherical cavity}

A spherical cavity of radius $a$ is subjected to a uniform pressure $\bar{p}$. Its boundary $\partial \Omega=\Sigma$ is symmetric with respect to the three coordinate planes, i.e. is invariant under the symmetry group $\mathcal{S}=P_{3}$, whose order is $n=8$ and whose irreducible representations (real-valued and of degree one) are given in table $\mathrm{X}$; this is obviously a case of complete symmetry, i.e. $\Gamma=\emptyset$. This choice of symmetry group is clearly not optimal: even disregarding groups of infinite order, like that of all rotations about an axis, the (non-abelian) symmetry group of the cube, of order 48 , could have been chosen instead. The symmetry cell $C$ is taken as the octant with positive coordinates. Four meshes (A,B,C,D) have been used, with $C$ being modelled with $M=3,12,48,108$ 8-noded elements respectively. The mesh parameters and 


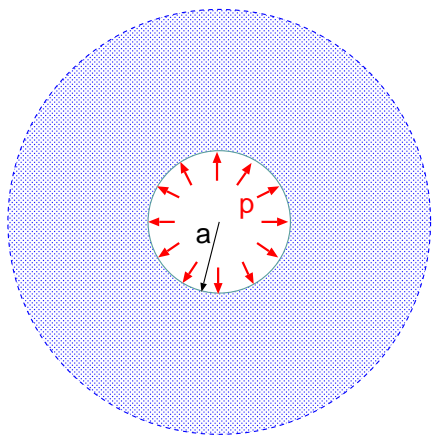

Figure 2. Example 1: geometry.

CPU timing results are given in table I, while table II displays the $L^{2}$ relative errors on the unknown density $\boldsymbol{\varphi}$ with respect to its exact value $\boldsymbol{\varphi}_{\mathrm{ex}}=3(1-\nu) \bar{p} a /(2 E) \boldsymbol{e}_{r}$. The block-diagonalization of $\boldsymbol{B}$ produces eight blocks, whose sizes are given in Table VIII.

\begin{tabular}{|l|rrrrrrrr|}
\hline Mesh & $M$ & $n N$ & $T_{I, S}(\mathrm{~s})$ & $T_{I, N}(\mathrm{~s})$ & $T_{R, S}(\mathrm{~s})$ & $T_{R, N}(\mathrm{~s})$ & $\tau_{I} / \tau_{I}^{e}$ & $\tau_{R} / \tau_{R}^{e}$ \\
\hline $\mathrm{A}$ & 3 & 222 & 0.48 & 2.69 & $<0.01$ & 0.02 & 1.43 & - \\
$\mathrm{B}$ & 12 & 870 & 2.82 & 19.5 & 0.07 & 0.83 & 1.16 & 5.40 \\
$\mathrm{C}$ & 48 & 3462 & 27.8 & 213. & 1.23 & 33.1 & 1.04 & 2.38 \\
$\mathrm{D}$ & 108 & 7782 & 127 & 981. & 8.85 & 340 & 1.04 & 1.67 \\
\hline
\end{tabular}

Table I. Example 1: mesh parameters, CPU times with (S) and without (N) exploiting geometrical symmetry, ratios of actual to expected gains in integration (I) and solution (S) CPU times.

\begin{tabular}{|l|rrrr|}
\hline Mesh & A & B & C & D \\
\hline$L^{2}$ error & $9.8(-3)$ & $1.2(-3)$ & $1.3(-4)$ & $9.4(-5)$ \\
\hline
\end{tabular}

Table II. Example 1: $L^{2}$ relative error on the unknown density $\varphi$.

\subsection{Example 2: round cylindrical bar with a penny-shaped central crack}

A round cylindrical bar occupying the region $\left(\Omega=\left\{-5 a \leq x \leq 5 a, y^{2}+z^{2} \leq a\right\}\right)$ is subjected on both its end surfaces $x= \pm 5 a$ to either a tensile load $\overline{\boldsymbol{p}}= \pm p_{0} \boldsymbol{e}_{x}$ or a flexural load $\overline{\boldsymbol{p}}= \pm\left(4 M_{0} z / 3 a^{4}\right) \boldsymbol{e}_{x}$. The external (cylindrical) boundary $\Sigma$ of this bar is symmetric with respect to the three coordinate planes, i.e. is invariant under the symmetry group $\mathcal{S}=P_{3}$. The symmetry cell $C$ is taken as the part of $\Sigma$ with positive coordinates. Inside the bar is a planar circular crack of radius $a$, in the plane normal to $\boldsymbol{e}_{x}$ and centered at the origin. This crack, which defines the surface $\Gamma$, happens to have $P_{2}$ symmetry but is treated as non-symmetric in the computation.

Two meshes have been considered: a coarse mesh (A) where $\Sigma$ and $\Gamma$ are modelled using $M_{1}=9$ and $M_{2}=18$ 8-noded elements, and a finer mesh (B) with $M_{1}=34$ and $M_{2}=408$ noded elements. The block-diagonalization of $\boldsymbol{B}$ produces eight blocks, whose sizes are given in Table VIII. The mesh parameters and CPU timing results are given in table III, while the relative 


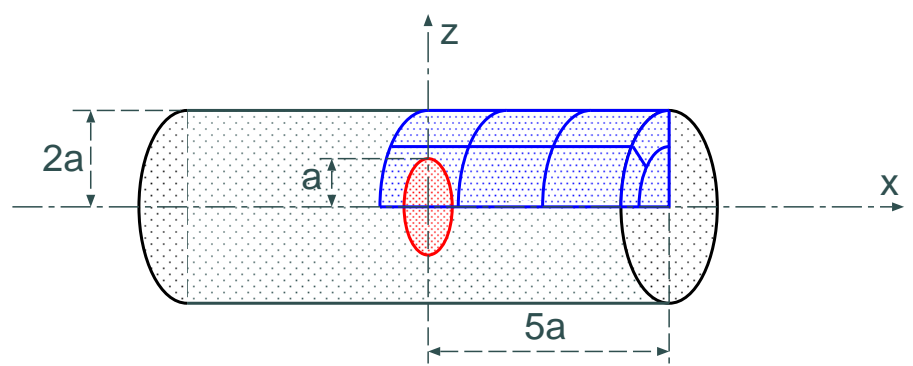

Figure 3. Example 2: geometry and boundary element mesh (A).

errors in the evaluation of the stress intensity factor $K_{I}$ using both meshes are displayed in table IV, based on comparisons with approximate solutions from [9] (for the flexural load and the present configuration, [9] provides the value of $K_{I}$ at the point $\left.(0, a, 0)\right)$. For the symmetry-reduced case, a preconditioned conjugate gradient (PCG) algorithm, where the preconditioner is the block-diagonal matrix Diag $\left(\left(\boldsymbol{B}_{\nu}^{a}\right)_{1 \leq \nu \leq m, 1 \leq a \leq d_{\nu}}, \boldsymbol{D}\right)$, has been used in addition to the direct algorithm described in section 8.3; the corresponding solution time appears within parentheses in Table III.

\begin{tabular}{|l|cccccccccc|}
\hline Mesh & $n N_{1}$ & $N_{2}$ & $M_{1}$ & $M_{2}$ & $T_{I, S}(\mathrm{~s})$ & $T_{I, N}(\mathrm{~s})$ & $T_{R, S}(\mathrm{~s})$ & $T_{R, N}(\mathrm{~s})$ & $\tau_{I} / \tau_{I}^{e}$ & $\tau_{R} / \tau_{R}^{e}$ \\
\hline $\mathrm{A}$ & 783 & 129 & 9 & 18 & 12.1 & 22.8 & $0.24(0.17)$ & 0.64 & 1.21 & 3.63 \\
$\mathrm{~B}$ & 2769 & 315 & 34 & 40 & 60.9 & 160 & $4.01(1.28)$ & 17.8 & 1.15 & 3.16 \\
\hline
\end{tabular}

Table III. Example 2: mesh parameters, CPU times with (S) and without (N) exploitation of partial geometrical symmetry, ratios of actual to expected gains in integration (I) and solution (S) CPU times.

\begin{tabular}{|l|rr|}
\hline Mesh & Tension & Flexion \\
\hline A & $1.5(-2)$ & $7.9(-3)$ \\
B & $2.2(-3)$ & $3.9(-3)$ \\
\hline
\end{tabular}

Table IV. Example 2: relative errors in the evaluation of the stress intensity factor $K_{I}$.

\subsection{Example 3: rectangular bar with a crack}

A rectangular bar occupying the region $(\Omega=\{-5 a \leq x \leq 5 a,-a \leq y \leq a,-a \leq z \leq a\})$ is subjected on both its end surfaces $x= \pm 5 a$ to a flexural load $\bar{p}= \pm p_{0} z / a e_{x}$. Again, the external boundary $\Sigma$ is invariant under the symmetry group $\mathcal{S}=P_{3}$. Inside the bar is a planar elliptical crack (semi-axes $0.67 a$ and $0.401 a$ ) centered at the point $(1.2 a,-0.2 a, 0.3 a)$ and inclided in a randomly chosen way, so that it is not invariant under any of the plane symmetries of $P_{3}$. Two meshes (A) and (B) have been considered, with $\Sigma$ modelled using (A) $M_{1}=28$ and (B) $M_{1}=69$ 8-noded elements, the crack being modelled using $M_{2}=40$ 8-noded elements in both cases. The mesh parameters and CPU timing results are given in table III. The solution times within parentheses in Table V correspond to the PCG algorithm as in example 2. The block-diagonalization of $\boldsymbol{B}$ produces eight blocks, whose sizes are given in Table VIII. 


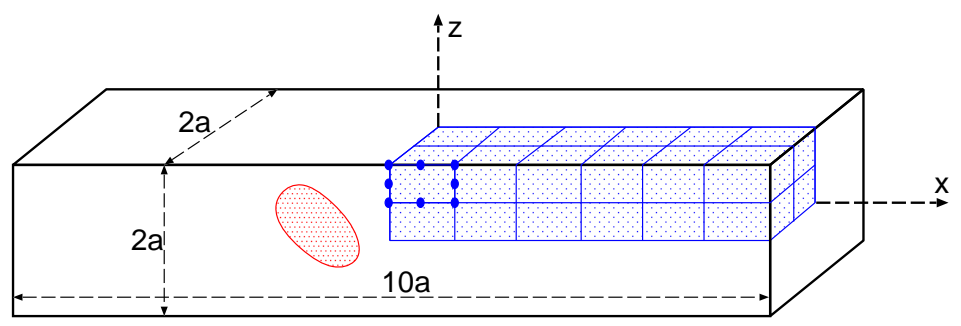

Figure 4. Example 3: geometry and boundary element mesh (A).

\begin{tabular}{|l|cccccccccc|}
\hline Mesh & $n N_{1}$ & $N_{2}$ & $M_{1}$ & $M_{2}$ & $T_{I, S}(\mathrm{~s})$ & $T_{I, N}(\mathrm{~s})$ & $T_{R, S}(\mathrm{~s})$ & $T_{R, N}(\mathrm{~s})$ & $\tau_{I} / \tau_{I}^{e}$ & $\tau_{R} / \tau_{R}^{e}$ \\
\hline A & 2022 & 129 & 28 & 18 & 27.7 & 97.2 & $1.15(0.65)$ & 8.88 & 1.26 & 3.00 \\
B & 4974 & 315 & 69 & 40 & 128.5 & 491 & $23 .(5.55)$ & 110 & 1.16 & 4.87 \\
\hline
\end{tabular}

Table V. Example 3: mesh parameters, CPU times with $(\mathrm{S})$ and without $(\mathrm{N})$ exploitation of partial geometrical symmetry, ratios of actual to expected gains in integration (I) and solution (S) CPU times.

\subsection{Example 4: bending of cracked prismatic beam with cross-shaped section}

A beam of length $10 a$ along the $x$-direction and with a cross-shaped section is considered, as depicted in Figure 5. It is subjected at both its end surfaces $x= \pm 5 a$ to a flexural load $\bar{p}= \pm p_{0} z / a \boldsymbol{e}_{x}$. The external boundary $\Sigma$ is invariant under the symmetry group $\mathcal{S}=D_{4} \times P_{x}$, where $D_{4}$ is the dihedral group of order 4 with $x$ as axis of rotation and $P_{x}$ is the group associated with the symmetry w.r.t. the

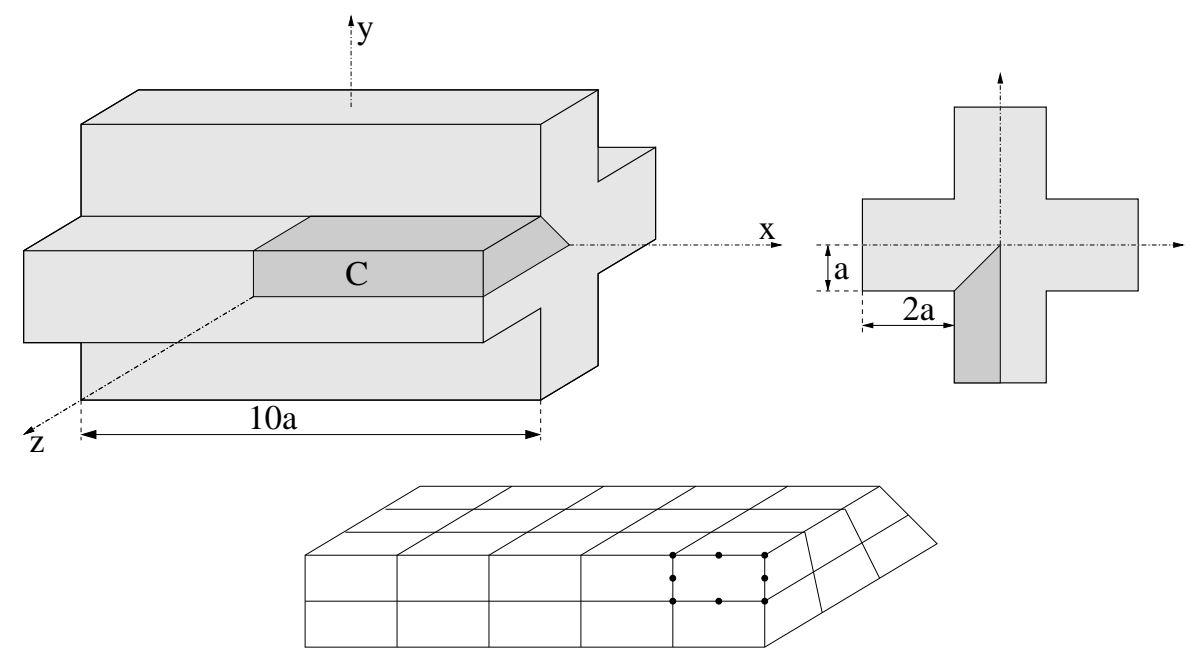

Figure 5. Example 4: geometry (top), section shape in $\left(x_{1}, x_{2}\right)$-plane (middle right) and BE mesh on the symmetry cell (bottom). 


\begin{tabular}{|l|cccccccccc|}
\hline Mesh & $n N_{1}$ & $N_{2}$ & $M_{1}$ & $M_{2}$ & $T_{I, S}(\mathrm{~s})$ & $T_{I, N}(\mathrm{~s})$ & $T_{R, S}(\mathrm{~s})$ & $T_{R, N}(\mathrm{~s})$ & $\tau_{I} / \tau_{I}^{e}$ & $\tau_{R} / \tau_{R}^{e}$ \\
\hline A & 4470 & 129 & 31 & 18 & 54.9 & 397 & $4.6(2.34)$ & 74.1 & 1.09 & 4.09 \\
B & 4470 & 315 & 31 & 40 & 75.4 & 438 & $14.1(3.35)$ & 81.9 & 1.17 & 5.16 \\
\hline
\end{tabular}

Table VI. Example 4: mesh parameters, CPU times with (S) and without (N) exploitation of partial geometrical symmetry, ratios of actual to expected gains in integration (I) and solution (S) CPU times.

plane $x=0 ; \mathcal{S}$ is a non-Abelian group of order $n=16$ whose representations are obtained from the Kronecker product of those of $D_{4}$ (Table XII) and $P_{x}$ (Table X). Inside the bar is a planar elliptical crack $\Gamma$ defined in the same way as in example 3. Two meshes (A) and (B) have been considered, with $\Sigma$ modelled using $M_{1}=31$ elements and $\Gamma$ with $M_{2}=18$ (A) or 40 (B) elements. The mesh parameters and CPU timing results are given in Table VI. The solution times within parentheses in Table VI correspond to the PCG algorithm as in example 2. The block-diagonalization of $\boldsymbol{B}$ produces 12 blocks (including two pairs of blocks of multiplicity 2), whose sizes are given in Table VIII.

\subsection{Cylinder with five regularly spaced channels}

A body of hollow cylindrical shape (length $12 a$ along the $x$-direction, inner and outer radii $4 a$ and $8 a$ ) with five parallel cylindrical channels of radius $a$ is considered (Figure 6). The channel axes are regularly spaced on a circle of radius $6 a$ in the $(y, z)$-plane (Figure 1), so that the boundary $\Sigma$ is invariant under the non-Abelian symmetry group $\mathcal{S}=D_{5} \times P_{x}$, of order $n=20$, where $D_{5}$ is the dihedral group of order 5 with $x$ as axis of rotation; the representations of $\mathcal{S}$ are obtained from the Kronecker product of those of $D_{5}$ (Table XI) and $P_{x}$ (Table X). Two meshes have been considered: mesh (A) for the above-described body (complete symmetry), with $\Sigma$ modelled using $M_{1}=30$ elements, and mesh (B) where in addition a planar elliptical crack (semi-axes $0.67 a$ and $0.401 a$ ) centered at the point $(2.3 a, 0.2 a, 5.8 a)$ and inclided in a randomly chosen way is embedded, its surface $\Gamma$ being modelled using $M_{2}=40$ elements. The channel with an axis defined by $(y, z)=(-6 a, 0)$ is loaded with a unit pressure, all other surfaces being traction-free. The mesh parameters and CPU
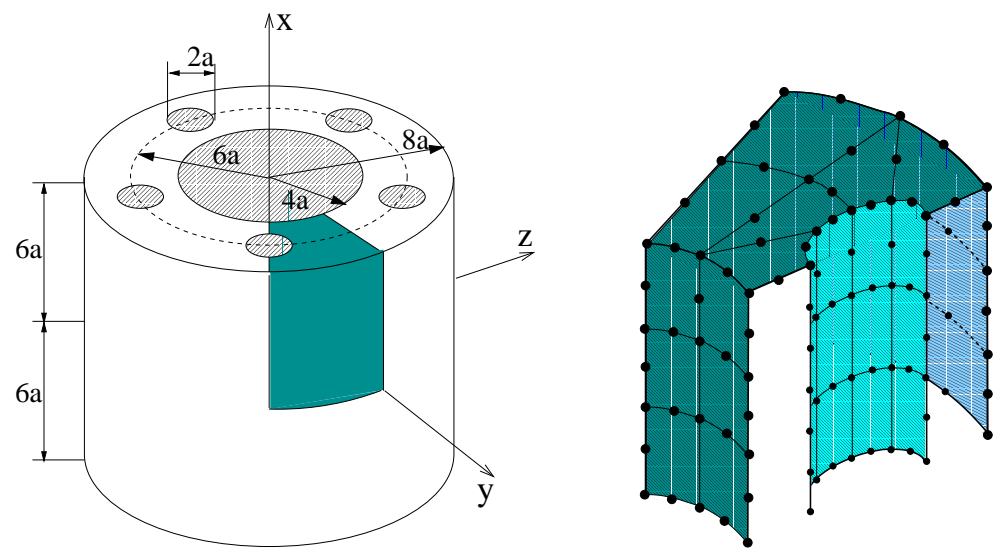

Figure 6. Example 5: geometry (left), BE mesh on the symmetry cell (right). 


\begin{tabular}{|l|cccccccccc|}
\hline Mesh & $n N_{1}$ & $N_{2}$ & $M_{1}$ & $M_{2}$ & $T_{I, S}(\mathrm{~s})$ & $T_{I, N}(\mathrm{~s})$ & $T_{R, S}(\mathrm{~s})$ & $T_{R, N}(\mathrm{~s})$ & $\tau_{I} / \tau_{I}^{e}$ & $\tau_{R} / \tau_{R}^{e}$ \\
\hline $\mathrm{A}$ & 5370 & - & 30 & - & 26.2 & 488 & 1.21 & 116 & 1.07 & 2.31 \\
$\mathrm{~B}$ & 5370 & 315 & 30 & 40 & 104 & 564 & $20.9(2.34)$ & 136 & 1.12 & 5.91 \\
\hline
\end{tabular}

Table VII. Example 5: mesh parameters, CPU times with (S) and without (N) exploitation of partial geometrical symmetry, ratios of actual to expected gains in integration (I) and solution (S) CPU times.

\begin{tabular}{|c|c|c|c|c|c|c|c|c|c|c|c|}
\hline & Mesh & $n N_{1}$ & & & & $\begin{array}{l}\text { 3lock } \\
\text { vith } d\end{array}$ & $\begin{array}{l}\text { izes } \\
=1)\end{array}$ & & & & $\begin{array}{l}\text { Block sizes } \\
\text { (with } d_{\nu}=2 \text { ) }\end{array}$ \\
\hline 1 & A & 222 & 33 & 30 & 30 & 26 & 30 & 26 & 26 & 21 & \\
\hline & B & 870 & 120 & 113 & 113 & 105 & 113 & 105 & 105 & 96 & \\
\hline & $\mathrm{C}$ & 3462 & 456 & 441 & 441 & 425 & 441 & 425 & 425 & 408 & \\
\hline & D & 7782 & 1008 & 985 & 985 & 961 & 985 & 961 & 961 & 936 & \\
\hline 2 & A & 783 & 93 & 90 & 84 & 80 & 84 & 80 & 74 & 69 & \\
\hline & B & 2769 & 328 & 321 & 311 & 303 & 311 & 303 & 293 & 284 & \\
\hline 3 & A & 2022 & 272 & 257 & 265 & 249 & 257 & 241 & 249 & 232 & \\
\hline & B & 4974 & 653 & 628 & 642 & 616 & 628 & 602 & 616 & 589 & \\
\hline 4 & & 4470 & 299 & 270 & 283 & 285 & 290 & 259 & 273 & 275 & $569 \quad 549$ \\
\hline 5 & & 5370 & 297 & 256 & 284 & 237 & & & & & $\begin{array}{llll}553 & 553 & 521 & 521\end{array}$ \\
\hline
\end{tabular}

Table VIII. Sizes (in numbers of DOFs) of the blocks $\boldsymbol{B}_{\nu}^{a}$ for the numerical examples of Section 10.

\begin{tabular}{|l|rrrr|}
\hline Step: & 1 & 2 & 3 & 4 \\
\hline CPU time recorded (s): & 0.85 & 17.5 & 2.2 & 0.04 \\
\hline CPU time estimated (s): & 0.85 & 2.99 & 1.95 & 0.04 \\
\hline
\end{tabular}

Table IX. Recorded and estimated execution times for steps 1 to 4 of the solution procedure with partial symmetry of section 8.3, using step 1 as a reference, for example 5 .

timing results are given in Table VII. The block-diagonalization of $\boldsymbol{B}$ produces 12 blocks (including four pairs of blocks of multiplicity 2), whose sizes are given in Table VIII. The solution time within parentheses in Table VII corresponds to the PCG algorithm as in example 2.

\subsection{Discussion}

In all the above numerical examples, the solution obtained with and without exploiting geometrical symmetry were for all practical purposes identical (their relative difference, in $L^{2}$ norm, was found to be lower than $10^{-5}$ in all cases). From Tables I, III, V, VI, VII, it is clear that the exploitation of partial geometrical symmetry provides quite substantial execution time savings. Moreover, in all those tables, the ratio $\tau_{I} / \tau_{I}^{e}$ is found to be quite close to unity, meaning that the actual gain $\tau_{I}$ in numerical integration time is in good agreement with the theoretical estimate $\tau_{I}^{e}$ given by (78). On the other hand, the ratio $\tau_{R} / \tau_{R}^{e}$ appears to be significantly (up to about five-fold) larger than unity, i.e. the actual gain $\tau_{R}$ in solution time does not agree with the theoretical estimate $\tau_{R}^{e}$ given by (81). To gain some insight into this discrepancy, let us for example consider the execution times spent into steps 1 to 4 outlined in section 8.3 for example 5: the recorded CPU times are displayed in Table IX and compared with the CPU times for steps 2, 3 and 4 predicted by the estimates of section 8.3 using as a reference the 
CPU time for step 1 (Choleski factorization of the blocks $\boldsymbol{B}_{\nu}^{a}$ ). Note that MATLAB provides computing time measurements in multiples of 0.01 second, so that small CPU times are not accurately recorded. From Table IX, step 2 as implemented appears to perform significantly slower than expected and to explain most of this timing discrepancy. We interpret this as caused by the MATLAB implementation: step 2 is implemented in the form $\left[\boldsymbol{Z}_{\nu}^{a}\right]=\left[\boldsymbol{R}_{\nu}^{a}\right]^{\mathrm{T}} \backslash\left[\boldsymbol{C}_{\nu}^{a}\right]$ using the MATLAB ' $\backslash$ ' (backslash) operator, which appears not to perform optimally on systems of equations with triangular matrices. We would expect actual gains $\tau_{R}$ to be much closer to their theoretical estimate $\tau_{R}^{e}$ in other implementations (e.g. using FORTRAN or C/C++). Even for the simpler case of complete symmetry, the ratio $\tau_{R} / \tau_{R}^{e}$ is not close to unity, and the reason is similar. For example, in example 1 with mesh (D), the solution times with and without symmetry exploitation are $5.81+2.99 \mathrm{~s}$ and $314+24.6 \mathrm{~s}$, respectively, where the time consumed by the Choleski factorization and the backsubstitutions are distinguished, in that order. One sees that the ratio of Choleski factorization times is $5.81 / 314 \approx 1 / 54$, quite closer to the theoretical value $1 / 64$, but also that the backsubstitution times are very high compared to the Choleski factorization times. Again, the discrepancy between actual and expected gains in solution time appears to be due to the fact that solving triangular systems of equations using the MATLAB built-in functions is relatively inefficient. Finally, in partial symmetry cases, the blocks $\boldsymbol{B}_{\nu}^{a}$ and $\boldsymbol{D}$ define a natural preconditioner for the conjugate gradient method, which is seen here to perform significantly faster than the direct solver.

\section{CONCLUSION}

In this paper, a systematic approach for the exploitation of geometrical symmetries in SGBEM formulations has been presented. The formulation has been expounded in detail for the relatively simple case of Neumann elastostatic boundary-value problems, but can be extended in a quite straightforward way to the SGBEM formulations of more general boundary-value problems, including complex-valued problems such as those arising in frequency-domain elastodynamics. The symmetry assumptions concern the geometrical shape of the boundary, the material properties (e.g. elasticity moduli) and the type of boundary conditions. On the other hand, the values of the quantities prescribed on the boundary are arbitrary, i.e. are not restricted by symmetry.

Another important result of this investigation is the fact that the approach is applicable in the case of partial symmetry in addition to the usual case of complete symmetry. This provides substantial savings in computing time and memory requirements when $\Gamma$ is 'small' (in terms of the number of DOFs involved). This is for instance the case for externally symmetric bodies containing holes, cracks or other defects of arbitrary shape and location. This investigation is thus expected to be highly beneficial to some computationally intensive problems like defect identification in complex bodies exhibiting external geometrical symmetry.

The strategy, including a systematic treatment of the constraints that the unknowns and trial functions must satisfy on the boundary of the symmetry cell, is fully general, i.e. is applicable to any finite group of isometries of the physical space $\mathbb{R}^{2}$ or $\mathbb{R}^{3}$. Some of the numerical examples presented in this paper concern complicated (and in particular non-Abelian) symmetry groups, with unsymmetric loadings, and the corresponding symmetry-exploiting formulations would be very difficult to obtain otherwise.

A number of extensions and generalizations are left out for future investigation. The most significant ones concern problems with mixed boundary conditions, which in principle does not raise new difficulties but requires a somewhat more involved implementation, and cases where both $\Sigma$ and $\Gamma$ have symmetry groups $\mathcal{S}^{1}, \mathcal{S}^{2}$, with $\mathcal{S}^{1,2}=\mathcal{S}^{1} \cup \mathcal{S}^{2}$ a non-trivial subgroup of $\mathcal{S}^{1}$ and $\mathcal{S}^{2}$, where the symmetry-exploiting formulation itself warrants further investigation. 


\section{REFERENCES}

1. Allgower, E. L., Georg, K., Miranda, R., Tausch, J. Numerical Exploitation of Equivariance. Z. Angew. Math. Mech., 78, 795-806 (1998).

2. Bonnet, M. On the use of geometrical symmetry in the boundary element methods for 3D elasticity. In C.A. Brebbia (ed.), Boundary element technology VI, pp. 185-201. Comp. Mech. Publ., Southampton / Elsevier, Southampton, Boston (1991).

3. Bonnet, M., Maier, G., Polizzotto, C. Symmetric Galerkin boundary element methods. Appl. Mech. Rev., 51, 669-704 (1998).

4. Bossavit, A. Symmetry, groups and boundary value problems : a progressive introduction to noncommutative harmonic analysis of partial differential equations in domains with geometrical symmetry. Comp. Meth. in Appl. Mech. Engng., 56, 167-215 (1986).

5. Eringen, A. C., Suhubi, E. S. Elastodynamics (vol II - linear theory). Academic Press (1975).

6. LoBry, J., BRoche, CH. Geometrical symmetry in the boundary element method. Engng. Anal. with Bound. Elem., 14, 229-238 (1994)

7. NeDELEC, J. C. Integral equations with non integrable kernels. Integral equations and operator theory, 5, 562-572 (1982).

8. SERRE, J. P. Linear representations of finite groups. Springer-Verlag (1977).

9. G. C. SIH. Handbook of stress-intensity factors. Lehigh University, Bethleem, Pennsylvania, USA, (1973).

10. Stroud, A. H. Approximate calculation of multiple integrals. Prentice-Hall (1971).

11. VINBERG, E. B. Linear representations of groups. Birkhäuser (1989).

\section{APPENDIX}

\section{PROOF OF THE DECOMPOSITIONS (26) and (49)}

For any $\boldsymbol{v} \in \mathcal{U}_{\Sigma}$, using definition (48) and changing the summation order, one has:

$$
\sum_{\nu=1}^{n} \sum_{a=1}^{d_{\nu}} \boldsymbol{P}_{\nu}^{a a} \boldsymbol{v}=\sum_{t \in \mathcal{S}}\left\{\sum_{\nu=1}^{n} \sum_{a=1}^{d_{\nu}} \frac{d_{\nu}}{n} \rho_{\nu}^{a a}(t)\right\} t^{-1} \boldsymbol{v}(t \boldsymbol{x})
$$

Moreover, the dual orthogonality (47) and the property $\left(44_{2}\right)$ imply that:

$$
\sum_{\nu=1}^{n} \sum_{a=1}^{d_{\nu}} \frac{d_{\nu}}{n} \rho_{\nu}^{a a}(t)=\sum_{\nu=1}^{n} \sum_{a, b=1}^{d_{\nu}} \frac{d_{\nu}}{n}\left[\rho_{\nu}^{a b}\right]^{\star}(t) \rho_{\nu}^{a b}(I)=\delta_{t I}
$$

Inserting this result into (87) yields the desired result (49). The decomposition (26) is then obtained as a special case of (49) where all representations are of degree 1.

\section{IRREDUCIBLE REPRESENTATIONS OF SOME SYMMETRY GROUPS}

Plane symmetry. The irreducible representations for the groups $P_{1}=\left\{I, s_{1}\right\}, P_{12}=$ $\left\{I, s_{1}, s_{2}, s_{1} s_{2}\right\}$ and $P_{123}=\left\{I, s_{1}, s_{2}, s_{1} s_{2}, s_{3}, s_{1} s_{3}, s_{2} s_{3}, s_{1} s_{2} s_{3}\right\}$ (where $s_{i}$ denotes the symmetry w.r.t. the plane $x_{i}=0$ ) are defined in Table X. In fact, since the two- and three-plane symmetry groups are Cartesian products of one-plane symmetry groups (e.g. $P_{13}=P_{1} \times P_{3}$ ), their irreducible representations are Kronecker products of those of the corresponding one-plane groups.

Cyclic symmetry. The irreducible representations for the groups $C_{n}=\left\{\mathrm{I}, r, r^{2}, \ldots, r^{n-1}\right\}$ (where $r$ is a rotation of angle $2 \pi / n$, with $2 \leq n$ ) are defined by:

$$
\rho_{\nu}(r)=\exp (2 i \pi \nu / n) \quad(\nu=0, \ldots, n-1)
$$


Dihedral groups $\mathcal{S}=D_{m}$. Let $\Delta$ denote a line in $\mathbb{R}^{3}$ and $\Sigma$ a plane containing $\Delta$. The dihedral group $D_{m}(m \geq 3)$ is generated by the symmetry $s$ w.r.t. $\Sigma$ and the $2 \pi / m$ rotation $r$ around $\Delta$, i.e. $D_{m}=C_{n} \cup s C_{m}$ (where $s C_{m}=\left\{s t, t \in C_{m}\right\}$ ). The dihedral group $D_{3}$ is the smallest non-Abelian symmetry group. The irreducible representations of $D_{n}$ have distinct structures according to whether $m$ is odd (see Table XI) or even (see Table XII).

\begin{tabular}{|c|cc|}
\hline$P_{1}$ & $\mathrm{I}$ & $s_{1}$ \\
\hline$\rho_{1}$ & +1 & +1 \\
\hline$\rho_{2}$ & +1 & -1 \\
\hline
\end{tabular}

\begin{tabular}{|c|cccc|}
\hline$P_{2}$ & $\mathrm{I}$ & $s_{1}$ & $s_{2}$ & $s_{1} s_{2}$ \\
\hline$\rho_{1}$ & +1 & +1 & +1 & +1 \\
\hline$\rho_{2}$ & +1 & -1 & +1 & -1 \\
\hline$\rho_{3}$ & +1 & +1 & -1 & -1 \\
\hline$\rho_{4}$ & +1 & -1 & -1 & +1 \\
\hline
\end{tabular}

\begin{tabular}{|c|cccccccc|}
\hline$P_{3}$ & $\mathrm{I}$ & $s_{1}$ & $s_{2}$ & $s_{3}$ & $s_{2} s_{3}$ & $s_{1} s_{3}$ & $s_{1} s_{2}$ & $s_{1} s_{2} s_{3}$ \\
\hline$\rho_{1}$ & +1 & +1 & +1 & +1 & +1 & +1 & +1 & +1 \\
\hline$\rho_{2}$ & +1 & +1 & +1 & -1 & -1 & -1 & +1 & -1 \\
\hline$\rho_{3}$ & +1 & -1 & +1 & +1 & +1 & -1 & -1 & -1 \\
\hline$\rho_{4}$ & +1 & -1 & +1 & -1 & -1 & +1 & -1 & +1 \\
\hline$\rho_{5}$ & +1 & +1 & -1 & +1 & -1 & +1 & -1 & -1 \\
\hline$\rho_{6}$ & +1 & +1 & -1 & -1 & +1 & -1 & -1 & +1 \\
\hline$\rho_{7}$ & +1 & -1 & -1 & +1 & -1 & -1 & +1 & +1 \\
\hline$\rho_{8}$ & +1 & -1 & -1 & -1 & +1 & +1 & +1 & -1 \\
\hline
\end{tabular}

Table X. Irreducible representations for plane symmetries with respect to one, two and three coordinate planes (respective orders $n=2,4,8$ ).

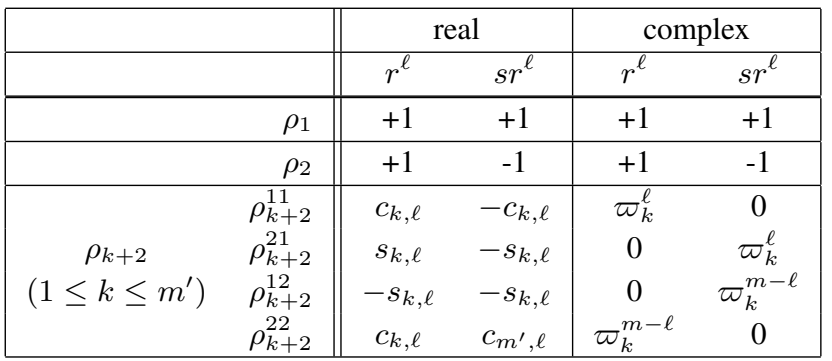

$(0 \leq \ell \leq m-1)$

Table XI. Two equivalent complete sets of irreducible representations for dihedral symmetry $\mathcal{S}=D_{m}(m$ odd, with $m=2 m^{\prime}+1, m^{\prime} \geq 1$ : real-valued representations (left, with $c_{k, \ell}=\cos 2 k \ell \pi / m$ and $s_{k}=\sin 2 k \ell \pi / m$ ) and complex-valued representations (right, with $\varpi_{k}=\exp (2 i k \pi / m)$ ).

\begin{tabular}{|cc||cc|cc|}
\hline \multicolumn{1}{|c||}{} & \multicolumn{2}{c|}{ real } & \multicolumn{2}{c|}{ complex } \\
\hline & $r^{\ell}$ & $s r^{\ell}$ & $r^{\ell}$ & $s r^{\ell}$ \\
\hline$\rho_{1}$ & +1 & +1 & +1 & +1 \\
\hline$\rho_{2}$ & +1 & -1 & +1 & -1 \\
\hline$\rho_{3}$ & $(-1)^{\ell}$ & $(-1)^{\ell}$ & $(-1)^{\ell}$ & $(-1)^{\ell}$ \\
\hline & $\rho_{4}$ & $(-1)^{\ell}$ & $(-1)^{\ell+1}$ & $(-1)^{\ell}$ & $(-1)^{\ell+1}$ \\
\hline & $\rho_{k+2}^{11}$ & $c_{k, \ell}$ & $-c_{k, \ell}$ & $\varpi_{k}^{\ell}$ & 0 \\
$\rho_{k+4}$ & $\rho_{k+2}^{21}$ & $s_{k, \ell}$ & $-s_{k, \ell}$ & 0 & $\varpi_{k}^{\ell}$ \\
& $\rho_{k+2}^{12}$ & $-s_{k, \ell}$ & $-s_{k, \ell}$ & 0 & $\varpi_{k}^{m-\ell}$ \\
& $\rho_{k+2}^{22}$ & $c_{k, \ell}$ & $c_{m^{\prime}, \ell}$ & $\varpi_{k}^{m-\ell}$ & 0 \\
\hline
\end{tabular}

$$
(0 \leq \ell \leq m-1)
$$

Table XII. Two equivalent complete sets of irreducible representations for dihedral symmetry $\mathcal{S}=D_{m}(m$ even, with $m=2 m^{\prime}+2, m^{\prime} \geq 1$ : real-valued representations (left, with $c_{k, \ell}=\cos 2 k \ell \pi / m$ and $s_{k}=\sin 2 k \ell \pi / m$ ) and complex-valued representations (right, with $\varpi_{k}=\exp (2 i k \pi / m)$ ). 